\title{
Can modular strategies simplify neural control of multidirectional human locomotion?
}

Karl E. Zelik, Valentina La Scaleia, Yuri P. Ivanenko and Francesco Lacquaniti

J Neurophysiol 111:1686-1702, 2014. First published 15 January 2014; doi:10.1152/jn.00776.2013

You might find this additional info useful...

This article cites 68 articles, 34 of which can be accessed free at:

/content/111/8/1686.full.html\#ref-list-1

This article has been cited by 1 other HighWire hosted articles

Modular organization across changing task demands in healthy and poststroke gait

Rebecca L. Routson, Steven A. Kautz and Richard R. Neptune

PHY2, June 1, 2014; 2 (6): .

[Abstract] [Full Text] [PDF]

Updated information and services including high resolution figures, can be found at:

/content/111/8/1686.full.html

Additional material and information about Journal of Neurophysiology can be found at: http://www.the-aps.org/publications/jn

This information is current as of December 3, 2014.

Journal of Neurophysiology publishes original articles on the function of the nervous system. It is published 12 times a year (monthly) by the American Physiological Society, 9650 Rockville Pike, Bethesda MD 20814-3991. Copyright @ 2014 by the American Physiological Society. ISSN: 0022-3077, ESSN: 1522-1598. Visit our website at http://www.the-aps.org/. 


\title{
Can modular strategies simplify neural control of multidirectional human locomotion?
}

\author{
Karl E. Zelik, ${ }^{1}$ Valentina La Scaleia, ${ }^{2}$ Yuri P. Ivanenko, ${ }^{1}$ and Francesco Lacquaniti ${ }^{1,2,3}$ \\ ${ }^{1}$ Laboratory of Neuromotor Physiology, IRCCS Santa Lucia Foundation, Rome, Italy; ${ }^{2}$ Department of Systems Medicine, \\ University of Rome Tor Vergata, Rome, Italy; and ${ }^{3}$ Centre of Space Bio-medicine, University of Rome Tor Vergata, \\ Rome, Italy
}

Submitted 30 October 2013; accepted in final form 14 January 2014

\begin{abstract}
Zelik KE, La Scaleia V, Ivanenko YP, Lacquaniti F. Can modular strategies simplify neural control of multidirectional human locomotion? J Neurophysiol 111: 1686-1702, 2014. First published January 15, 2014; doi:10.1152/jn.00776.2013.—Each human lower limb contains over 50 muscles that are coordinated during locomotion. It has been hypothesized that the nervous system simplifies muscle control through modularity, using neural patterns to activate muscles in groups called synergies. Here we investigate how simple modular controllers based on invariant neural primitives (synergies or patterns) might generate muscle activity observed during multidirectional locomotion. We extracted neural primitives from unilateral electromyographic recordings of 25 lower limb muscles during five locomotor tasks: walking forward, backward, leftward and rightward, and stepping in place. A subset of subjects also performed five variations of forward (unidirectional) walking: self-selected cadence, fast cadence, slow cadence, tiptoe, and uphill ( $20 \%$ incline). We assessed the results in the context of dimensionality reduction, defined here as the number of neural signals needing to be controlled. For an individual task, we found that modular architectures could theoretically reduce dimensionality compared with independent muscle control, but we also found that modular strategies relying on neural primitives shared across different tasks were limited in their ability to account for muscle activations during multi- and unidirectional locomotion. The utility of shared primitives may thus depend on whether they can be adapted for specific task demands, for instance, by means of sensory feedback or by being embedded within a more complex sensorimotor controller. Our findings indicate the need for more sophisticated formulations of modular control or alternative motor control hypotheses in order to understand muscle coordination during locomotion.
\end{abstract}

neural control; modularity; human locomotion; EMG; muscle synergies

CONTROLLING EACH of the numerous muscles of the lower limb individually seems to pose a computational challenge for the sensorimotor control system (d'Avella et al. 2003; Giszter et al. 2010), which must generate different muscle activation profiles depending on the type and direction of locomotion. It has been proposed that a modular strategy might simplify intermuscular coordination by enabling the nervous system to control muscles in groups (called synergies), which could reduce dimensionality, that is, reduce the number of neural activation outputs controlled (d'Avella et al. 2003; Ting and Macpherson 2005; Tresch et al. 1999). This strategy might provide a parsimonious neural control solution that still maintains adequate flexibility to generate task-specific muscle activity. Theoretically, the nervous system could implement such a control

Address for reprint requests and other correspondence: K. E. Zelik, Laboratory of Neuromotor Physiology, IRCCS Santa Lucia Foundation, Via Ardeatina 306, 00179 Rome, Italy (e-mail: k.zelik@hsantalucia.it). strategy in a multitude of ways, but there is no consensus on the basic building blocks or the general organization of neural modularity (Alessandro et al. 2013; Lacquaniti et al. 2012; Tresch and Jarc 2009). Thus we sought to explore how various modular architectures, if used by the nervous system, might generate muscle activity profiles for different locomotor tasks.

Neural modularity is characterized by neural primitives (also called modules) that can be flexibly recombined to generate muscle activations for disparate motor behaviors. While this concept of modularity has been formulated in various ways (d'Avella et al. 2003; Delis et al. 2014; Drew et al. 2008; Ivanenko et al. 2004; Ting and Macpherson 2005), the simplest architectures one might think of would involve invariant neural primitives. For instance, motor behaviors could be performed by activating a set of invariant muscle synergies with patterns (waveforms) of different shape and timing. Alternatively, a controller might reuse fixed temporal patterns but modulate which groups of muscle synergies they activate and in what proportion. Neural primitives might also be more complex, and various alternatives have been proposed, for example, relying on spatiotemporally varying modules. These possibilities constitute a portion of the candidate primitives operating at the level of individual muscles, but neural modularity may also (or instead) be realized at the level of motoneurons, interneurons, spinal segments, individual joints, or cortical/subcortical circuitry (Lacquaniti et al. 2013; Tresch and Jarc 2009).

Modularity could also be organized in diverse ways, affecting the degree to which neural primitives are shared between tasks (d'Avella and Bizzi 2005; Cheung et al. 2005; Chhabra and Jacobs 2006; Flanders 2011; Lewicki and Sejnowski 2000; Tresch and Jarc 2009). Organizational structures might be based principally on shared primitives, task-specific primitives, or a combination of each. Also, neural primitives might be adapted by task-specific sensory feedback, or be part of an overcomplete representation of movement, in which a vast array of modules might be neurally encoded and then selectively activated. Furthermore, perspectives vary greatly as to how much of movement might be controlled by modular versus nonmodular processes.

The breadth of proposed modular architectures (i.e., neural building blocks and how they are organized) can hardly be tested in any single study. Nevertheless, a rigorous and systematic investigation of a subset of these modular architectures may yield useful neurophysiological and methodological insights. Here we investigate how simple modular controllers might generate muscle activations during multidirectional lo- 
comotion and interpret the results in the context of dimensionality reduction.

\section{METHODS}

We recorded muscle activation profiles from different locomotor tasks. We extracted modules (synergies or patterns) from unilateral electromyography (EMG) recordings, using standard nonnegative matrix factorization (NNMF) methods, first assuming task-specific libraries of modules and next assuming a single, shared library. For each control architecture we then computed the minimum number of modules needed to reconstruct the recorded EMGs during performance of a task and the total number of modules across all tested tasks. The number of modules needed for a single task was then compared with the number of recorded muscles activated during that task, in order to investigate the dimensionality of control compared with controlling each muscle independently. To keep this study focused and computationally tractable, we limited the scope to invariant primitives (synergies or patterns) organized in simple architectures.

\section{Experimental Protocol}

We recorded surface EMG and foot motion for eight participants (4 men, 4 women; $25.6 \pm 2.6 \mathrm{yr}$ old, $1.78 \pm 0.11 \mathrm{~m}, 76 \pm 16 \mathrm{~kg})$ during different locomotor tasks. In the main experiment (performed by all 8 participants), these tasks were walking forward $(4 \mathrm{~km} / \mathrm{h})$, walking backward $(3.5 \pm 0.5 \mathrm{~km} / \mathrm{h})$, walking sideways to the left and to the right $(3 \mathrm{~km} / \mathrm{h})$, and stepping in place. Sideways walking was performed in a manner such that the trailing limb never crossed ahead of the leading limb. Some participants were unable to walk at the desired backward speed of $4 \mathrm{~km} / \mathrm{h}$ and therefore were tested at $3 \mathrm{~km} / \mathrm{h}$. In half the subjects, the stepping in place cadence was fixed to match the step frequency of forward walking (via a metronome), but this frequency matching was observed to have little effect on the results, since EMGs for all conditions were eventually normalized to a single gait cycle. These multidirectional tasks were chosen to represent biomechanically distinct movements that might share common neural control pathways (Lamb and Yang 2000) and that could be performed at steady state with sufficiently low intensity not to dislodge surface electrodes. We also collected additional data on unidirectional walking tasks to assess whether our results were specific to multidirectional gait or potentially generalizable to other forms of locomotion. A subset of subjects $(n=4)$ performed another experiment involving five variations of forward walking at $4 \mathrm{~km} / \mathrm{h}$ : self-selected cadence, fast step cadence (as enforced by a metronome), slow step cadence, tiptoe, and incline (20\% grade).

Prior to data collection the subjects were trained on each task, allowing them time to acclimate to the various walking conditions on the treadmill. All tasks were performed barefoot on a standard treadmill for $40 \mathrm{~s}$, from which we analyzed the middle $30 \mathrm{~s}$. The protocol was approved by the Ethics Committee of the Santa Lucia Institute, and all subjects gave informed consent prior to participation.

\section{EMG Recordings}

Twenty five muscles were recorded from the right side of each subject. These included 3 muscles from the lower back and buttocks (erector spinae at $\mathrm{L}_{2}$ level, gluteus maximus, gluteus medius), 11 from the thigh (vastus medialis, vastus lateralis, rectus femoris, biceps femoris long head and short head, semitendinosus, sartorius, iliopsoas, adductor longus, adductor magnus, tensor fasciae latae), 6 from the shank (medial and lateral gastrocnemius, soleus, tibialis anterior, peroneus longus and brevis), and 5 from the foot (extensor hallucis brevis, extensor digitorum brevis, flexor digitorum brevis, extensor hallucis longus, flexor digitorum/hallucis longus). The flexor digito- rum longus and flexor hallucis longus activations were indistinguishable in our surface EMG recordings and thus are reported together. Electrodes were placed based on recommendations from SENIAM (seniam.org), the European project on surface EMG. For muscles not included in SENIAM, the electrodes were placed by palpating muscles to locate the muscle belly and orienting the electrodes along the main direction of the fibers (Kendall 2005). The muscles recorded were limited to those that could be reliably located by palpation and were superficial enough to be monitored with surface EMG. All EMGs were recorded at $4,000 \mathrm{~Hz}$ with the Delsys Trigno Wireless System (Boston, MA) except the flexor digitorum brevis, which was recorded at $1,000 \mathrm{~Hz}$ with a synchronized Delsys Bagnoli System. This was necessary because of the recording site of flexor digitorum brevis (on the bottom of the foot), which required a lower-profile electrode (i.e., sensor thickness of 6 vs. $15 \mathrm{~mm}$ ). Because of the large number of muscles recorded and the challenge of recording from various foot muscles, some electrodes became partially or fully detached during testing, leading to unusable EMG signals. These EMGs were removed on a subject-specific basis. On average we analyzed 23.6 \pm 1.4 EMGs from each subject.

\section{Motion Capture}

Heel motion was tracked to identify gait cycles for each task. The position of the right heel (calcaneus) was recorded at $100 \mathrm{~Hz}$ with a nine-camera Vicon-612 motion capture system (Vicon, Oxford, UK), which was synchronized with the EMG acquisition. The motion of the heel was low-pass filtered at $12 \mathrm{~Hz}$ (zero-lag 3rd-order Butterworth) and resampled at the EMG sampling frequency. For all walking tasks, the beginning of the gait cycle was defined as the maximum forward excursion of the right heel, where forward refers to the direction of the treadmill. This generally occurred slightly before foot strike (typically $<5 \%$ of gait cycle). While foot strike is more commonly used in the literature to delineate the beginning of a gait cycle, we found forward excursion to be more robust across all the walking tasks. Ultimately, the event that signifies the start of the gait cycle is a subjective choice, the most important criterion being the repeatability that allows one to average across multiple strides. For stepping in place, we defined the start of the gait cycle as the time at which the vertical displacement of the right heel reached its minimum (an estimate of foot strike). These differences in definition of the start of the gait cycle introduced minor time shifts between tasks. Since all muscle EMGs for a single task shift together in time, this had no impact on extracting shared muscle synergies, which were assumed to be time invariant. The differences were, however, considered when extracting shared patterns, as detailed in Factorization below.

\section{EMG Processing}

EMG signals were processed with conventional filtering and rectifying methods. Flexor digitorum brevis EMGs were resampled to match the other EMG signals. Then the mean signals were removed from all raw EMGs. Next, filtering was performed: we applied a $30-\mathrm{Hz}$ high-pass filter, followed by a $50-\mathrm{Hz}$ notch filter (to remove power-line artifacts), then rectified the signal, and finally applied a $10-\mathrm{Hz}$ low-pass filter (all filters, zero-lag 3rd-order Butterworth). A few subjects exhibited artifacts in the foot and peroneus muscles, generally linked to foot strike and toe-off events. To remove these artifacts, high-pass filtering of these muscles was performed with a $150-\mathrm{Hz}$ cutoff frequency (rather than $30 \mathrm{~Hz}$ ). Informal tests confirmed that this artifact-removal filter had minimal effect on the shape of the muscle activation pattern. For all EMG envelopes, small negative values due to filtering overshoot were zeroed, a necessity for the subsequent NNMF analysis. We divided EMGs into gait cycles based on foot kinematics, then interpolated each stride to 1,000 time points, and finally averaged across gait cycles (individually for each subject and task). This yielded an $n \times m$ EMG matrix for each task, where $n$ 
equaled 1,000 time points and $m$ equaled the number of muscles analyzed. These processed and stride-averaged EMG envelopes (in $\mu \mathrm{V}$ ) were then normalized and analyzed for each subject individually, as detailed below.

\section{EMG Normalization}

We normalized EMGs by the maximum activation magnitude. Normalization was performed to account for the differences in microvolt magnitudes recorded between muscles. Each muscle EMG was normalized by its maximum activation magnitude, which we defined as the muscle's maximum EMG signal from either dynamic or quasi-static trials, detailed below. Thus all EMGs are reported on a scale from 0 to 1 , where 0 indicates that a muscle is inactive and 1 represents maximum activity.

Additional quasi-static trials were performed to establish the maximum voluntary EMG magnitude for each muscle. Before the experimental tasks, subjects performed a set of quasi-static maneuvers against manual resistance. These included flexing/extending/abducting the toes, plantarflexing/dorsiflexing/inverting/everting the ankle, flexing/extending the knee, flexing/extending/abducting/adducting the hip, and flexing the back. Each exercise was performed for $5 \mathrm{~s}$, during which subjects were instructed to perform maximal contractions. The EMGs were filtered as described previously. Across all quasi-static trials, we looked at a sliding 1-s window and computed the average EMG during each. The highest average EMG found during any 1-s window was defined as the maximum quasi-static activation magnitude. Similarly, maximum dynamic activation magnitude was defined for each muscle as the peak stride-averaged EMG across all five multidirectional tasks. The normalization constant for each muscle was then defined as the larger of the quasi-static and dynamic activation magnitudes.

During analysis, we also tested other types of EMG normalization, such as using only maximum dynamic activation magnitudes or scaling normalized EMG by muscle physiological cross-sectional area. In general, qualitative findings appeared insensitive to the exact procedure used for EMG normalization.

\section{Factorization}

We extracted neural control modules (synergies and patterns) from the stride-averaged EMG signals with NNMF (Lee and Seung 1999) similar to prior literature (Fig. 1A; Ivanenko et al. 2005; Tresch et al. 1999). Briefly, this is a signal decomposition method that tries to best describe a matrix of muscle EMGs by a linear combination of muscle synergies $\left(W_{\mathrm{i}}\right)$ and temporal activation patterns $\left(C_{\mathrm{i}}\right)$.

$$
\mathrm{EMG}=\sum_{i}^{k} C_{i} W_{i}+\text { error, } k \leq m
$$

A muscle synergy (also called simply "synergy") represents a set of weightings for muscles that are activated together. Specifically, the time-invariant vector $W_{i}$ indicates the proportional contribution of each individual muscle to the $i$ th synergy. Thus the muscle weightings in each synergy are assumed to scale together linearly. The activation pattern $C_{i}$ is a waveform (defined over a gait cycle) representing the neuromotor signal that modulates the excitation of its associated synergy. When the number of synergies and patterns $(k)$ is sufficient to reconstruct the EMGs, but still substantially smaller than the number of muscles commanded $(m)$, then this modular decomposition reveals a potential strategy for reducing the dimensionality of neural control. We performed four variants of this general factorization method to study simple modular architectures.

Variant I. Assuming task-dependent architectures (Fig. 1B), we extracted patterns and synergies from each task separately. The modules extracted from a task, which we call the task-dependent or task-specific library, represent the minimum set of fixed modules that is needed to adequately reconstruct EMGs during that task. The criteria used to define adequate EMG reconstruction, and thus to select the minimum number of modules, are detailed in the next section below. It was not possible to differentiate between task-dependent synergy and task-dependent pattern architectures since mathematically these represent identical solutions, although they may reflect modularity at different layers of the neural circuitry.

Variant II. For task-independent architectures (Fig. 1C), we extracted shared patterns and then shared synergies from multiple tasks. We performed this analysis in a cumulative fashion. First, EMGs from forward walking were factorized alone. This was identical to the task-dependent factorization. Then forward and backward walking were factorized together. Then forward, backward, and sideways walking (to the left) were factorized, and so forth, until all five tasks were ultimately factorized together. We defined the task-independent (shared) library of fixed patterns and the task-independent library of fixed synergies as those extracted from all five tasks together. To extract the library of shared synergies, the EMGs for each task were concatenated along the time dimension of the matrix, yielding a matrix with $(1,000 \times$ number of tasks) rows and (number of muscles) columns, where 1,000 indicates the number of time points per gait cycle. To extract the library of shared temporal patterns we concatenated EMGs along the muscle dimension of the matrix, yielding a
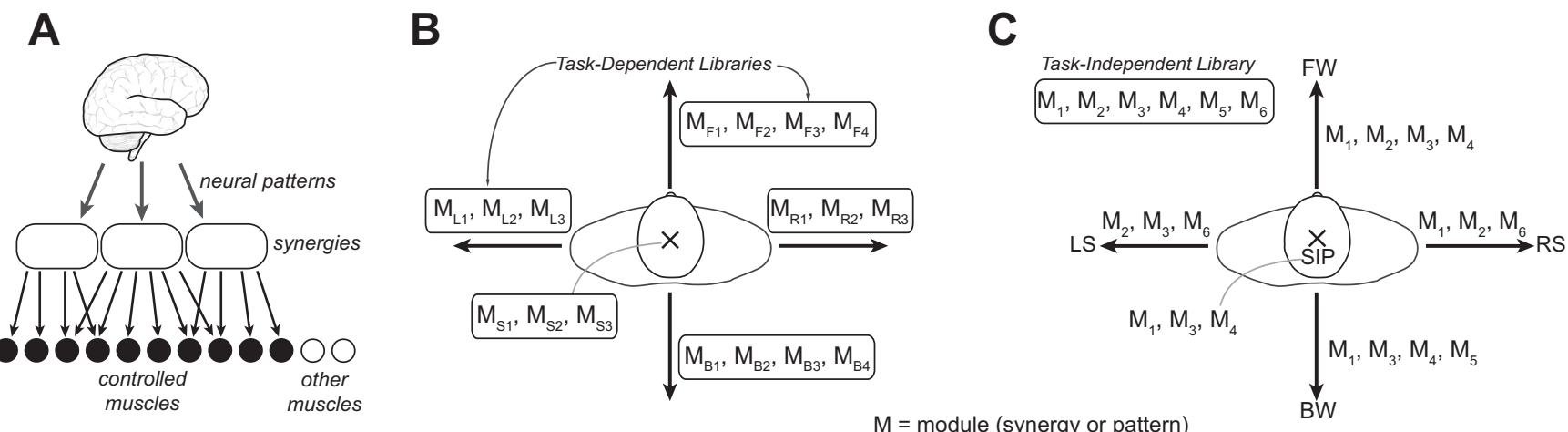

Fig. 1. Experimental design. A: dimensionality of modular control was evaluated by computing the modular control ratio (MCR), the number of neural primitives (patterns or synergies, also called modules) divided by the number of muscles activated (black circles) during a task. The example shown here of 3 primitives controlling 10 muscles yields an MCR $=0.3$, in other words, a 70\% reduction in dimensionality compared with controlling each muscle individually. For clarity, the central nervous system is depicted here by an image of the brain, although the primitives themselves may exist at either the spinal or the supraspinal level and may receive input from both feedforward and feedback pathways. We extracted primitives from multidirectional locomotion assuming 2 simplified models of modularity, task-dependent $(B)$ and task-independent $(C)$ architectures, where the modular primitives are designated M. The number of modules shown here for each task is arbitrary, and thus the examples in $B$ and $C$ are only intended for illustrative purposes. Experimental conditions studied were walking forward (FW), backward (BW), and sideways to the left (LS) and to the right (RS) and stepping in place (SIP). 
matrix with 1,000 rows and (number of muscles $\times$ number of tasks) columns. NNMF was then performed on these combined EMG matrices. Next, we found the subset of modules in each shared library that was needed to reconstruct the EMGs for each individual task. For example, a shared library might have 10 modules, but only 8 were needed to reconstruct forward walking EMGs. We calculated this subset of shared modules with a greedy search algorithm. Put simply, we systematically eliminated each shared module and then recomputed the estimated EMGs until we identified the smallest subset of modules that could still satisfy the EMG reconstruction criterion for a given task.

Variant III. We also tested a special instance of the task-independent architecture. We sought to answer the question: can the fixed synergies or patterns extracted from forward walking be used to reconstruct EMGs from the other modes of locomotion? If so, the modules contained in normal walking could be recombined to coordinate other locomotor tasks, an idea that has been suggested to simplify responses to perturbations during gait (Chvatal and Ting 2012). We therefore extracted muscle synergies $\left(W_{\mathrm{FW}}\right)$ from forward walking EMGs and then found the nonnegative least-squares solutions that mapped $W_{\mathrm{FW}}$ onto the EMG matrices from each other task. Next, we performed a similar extraction and mapping procedure using the neural patterns $\left(C_{\mathrm{FW}}\right)$ found during forward gait. We also allowed the extracted temporal patterns to shift together up to $\pm 10 \%$ of the stride to account for small differences in timing between tasks (Ivanenko et al. 2004), which might be due to how gait cycles were defined or differences in relative stance duration. Next, we estimated EMG waveforms assuming fixed $W_{\mathrm{FW}}$ and then fixed $C_{\mathrm{FW}}$, and we computed their correlations with the experimental data.

Variant $I V$. Finally, we explored task-independent architectures during variations of forward walking to determine whether modules might be shared among unidirectional locomotor tasks. A subset of participants $(n=4)$ performed five variations of forward walking at $4 \mathrm{~km} / \mathrm{h}$ : self-selected, fast step cadence (as enforced by a metronome), slow step cadence, tiptoe, and incline ( $20 \%$ grade). EMG processing and factorization were performed in identical fashion to the prior analyses (see variant II above), and we computed the minimum number of shared modules needed to reconstruct the EMGs.

\section{Evaluation Criteria}

We established a criterion to determine the minimum number of modules needed for each architecture to adequately reconstruct EMGs. Modular architectures were assessed by correlating their reconstructed estimates of EMG with the processed experimental recordings. The coefficient of determination $\left(R^{2}\right)$ was computed as the square of the centered Pearson correlation coefficient. To quantify the minimum number of synergies or patterns needed to adequately reconstruct the EMG data, we established an Individual-Muscle reconstruction criterion somewhat similar to the "local" criterion used in prior literature (Chvatal and Ting 2012; Roh et al. 2012). The reconstructed EMG waveform for each individual muscle was correlated with the corresponding experimental EMG. The IndividualMuscle criterion required that $90 \%$ of the Active muscle EMGs be reconstructed with $R^{2} \geq 0.8$, where Active muscles were defined as those whose peak EMG during a task surpassed 0.2 (20\% of the maximum activation magnitude). This evaluation criterion was an attempt to ensure that most individual muscle reconstructions were reasonable reflections of the empirical EMG recordings, while considering the biological and experimental noise and variability. The Active muscle distinction accounts for the fact that measurement variability may obscure EMGs at low activation levels, and thus it may be impractical to expect high reconstruction fidelity of these signals.

For comparison, we also included some results based on a Grouped-Muscle criterion, which has commonly been used in prior literature (Clark et al. 2010; Frere and Hug 2012; Ivanenko et al.
2005; Ting and Macpherson 2005). Grouped-Muscle evaluation involves correlating the entire matrix of experimental EMGs with the entire matrix of reconstructed EMGs, as opposed to comparing muscle waveforms individually. Grouped-Muscle or "global" criteria generally require the $R^{2}$ (centered Pearson correlation coefficient) or the variance accounted for (VAF, uncentered Pearson coefficient) of the overall EMG matrix reconstruction to be greater than some threshold (e.g., VAF $\geq 90 \%$ or $R^{2} \geq 0.9$ ). For comparison purposes in this study we used the $R^{2} \geq 0.9$ Grouped-Muscle criterion. Other variants of Grouped-Muscle criteria, such as adding modules until the increase in VAF is $<3-5 \%$ (Clark et al. 2010; Ivanenko et al. 2005), yield similar results. While such Grouped-Muscle criteria ensure that the muscles with the highest amplitude are reasonably reconstructed, we found that often many other muscle EMGs were poorly estimated. For instance, when using a Grouped-Muscle criterion to select the number of task-specific modules for forward walking we found that on average $40 \%$ of the individual EMGs were poorly reconstructed $\left(R^{2}<\right.$ 0.8 ), which would likely cause substantial degradation at the functional level of task performance (Alessandro et al. 2013; de Rugy et al. 2013). Therefore, we concluded that Grouped-Muscle criteria tend to underestimate the number of modules needed to reconstruct muscle activity during locomotion. Furthermore, we recognized that even Individual-Muscle evaluations could underestimate the number of neural modules needed if the "good enough" threshold were too low to ensure that individual EMGs were reasonably reconstructed, an issue addressed in Assumptions and Limitations below and in DISCUSSION.

\section{Modular Control Ratio}

For each task we defined the modular control ratio (MCR) as the minimum number of neural control modules needed divided by the number of muscles being controlled (Fig. 1A). During performance of a task there is a one-to-one correspondence between patterns and synergies. Thus MCR can also be thought of as the minimum number of patterns or synergies divided by the number of muscles being controlled. The lower the ratio, the greater the reduction in dimensionality compared with controlling each muscle individually. An MCR equal to 1 corresponds to the dimensionality of independently controlled muscles (i.e., no simplification of control). The benefit of using MCR is that by comparing the dimensionality of neural control directly to the dimensionality of muscle activations we can assess each modular architecture independently.

We estimated the numerator of the MCR as the minimum number of modules needed to reconstruct EMGs from each task. We estimated the denominator, the number of muscles being controlled, as the number of Active muscles per task, again defined as those muscles with peak EMG activation above $20 \%$ of maximum.

$$
\begin{aligned}
& \text { MCR }=\frac{\text { no. of modules }}{\text { no. of muscles controlled }} \\
& \approx \frac{\text { no. of modules needed to reconstruct Active muscle EMGs }}{\text { no. of Active muscles }}
\end{aligned}
$$

The benefit of this formulation is that it evaluates the neural controller's ability to generate the largest, and presumably most important, muscle activations. But the drawback is the need to set an arbitrary threshold to define which muscles are "Active."

Therefore, we also computed the MCR using an alternate, complementary estimation that does not require a distinction between "Active" and "Inactive" muscles. This alternate estimate inverts the approach used to identify modules. Rather than asking how many modules are needed to reconstruct the measured EMG signals, we formulate the question: given $N$ number of modules, how many muscle EMGs can be reconstructed? We refer to this alternate definition as $\mathrm{MCR}_{\mathrm{A}}$. For a task-dependent architecture, factorization was 
performed on each task using $N$ modules, where $N$ was varied from 3 to 9. Then the $\mathrm{MCR}_{\mathrm{A}}$ was computed for each value over this range. In this case, the number of modules was assumed to be $N$, and then the number of muscles controlled (modularly) was estimated as the number of EMGs that were adequately reconstructed $\left(R^{2} \geq 0.8\right)$ by those $N$ modules. Similarly, for task-independent architectures, factorization of the combined set of EMGs from all tasks was performed with $3 \leq N \leq 19$ factors, and $\mathrm{MCR}_{\mathrm{A}}$ s were computed. Here $N$ indicates the total size of the shared library (i.e., the number of modules needed to reconstruct all tasks). Therefore, the minimum number of control modules needed for each individual task was less than or equal to $N$. This number of modules per task was computed with a greedy search algorithm, similar to that described above. We systematically removed shared modules that did not increase the number of well-reconstructed EMGs, yielding the minimum subset of modules necessary for a given task. We used this number of modules as the numerator. The number of muscles controlled by the task-independent architecture (i.e., the denominator) was again estimated as the number of EMGs that were adequately reconstructed $\left(R^{2} \geq 0.8\right)$.

$$
\mathrm{MCR}_{\mathrm{A}}=\frac{\text { no. of modules }}{\text { no. of muscle EMGs reconstructed }}
$$

A benefit of computing $\mathrm{MCR}_{\mathrm{A}}$ is that it does not require a control strategy to reconstruct any preset percentage of muscle EMGs. However, as a consequence, some of the muscle activity that is reconstructed may be small in magnitude, and potentially less functionally relevant. Nevertheless, despite the limitations, the use of this metric in conjunction with the previous estimate of MCR provides some insight into dimensionality reduction, especially in instances when both metrics yield similar results.

To supplement the interpretation of $\mathrm{MCR}_{\mathrm{A}}$, which is defined for all values of $N$, we also computed the percentage of muscle EMGs that were well-reconstructed. Therefore, if $\mathrm{MCR}_{\mathrm{A}}$ was low for a given $N$, but only a small fraction of the EMGs could be reconstructed, then this would be clearly evident. To assess the sensitivity of this measure to the subset of muscles analyzed we also performed these computations on 1) the 20 nonfoot muscles and 2) a set of 12 muscles commonly recorded in the literature on modular control (gluteus maximus and medius, tensor fasciae latae, vastus medialis and lateralis, rectus femoris, biceps femoris, semitendinosus, tibialis anterior, medial and lateral gastrocnemius, and soleus).

\section{Statistics}

Statistical comparisons between the number of fixed synergies and the number of fixed patterns were made by paired $t$-tests with significance level 0.05 and Bonferroni-Holm correction for multiple comparisons. Adjusted $P$ values are reported. All EMG processing and factorizations were performed on a subject-specific basis. Summary measures in Figs. 2, 4, 5, 6, 7, and 9 were reported as intersubject means and standard deviations. Statistics were then computed on these summary measures.

\section{Assumptions and Limitations}

There are various limitations to inferring neural control strategies from EMG signals. While similar factorization methods have been used in the majority of studies on modular control (d'Avella and Bizzi 2005; Chvatal and Ting 2012; Ivanenko et al. 2004; Overduin et al. 2012; Ting and Macpherson 2005), these only provide an indirect indication of neural activity and are sensitive to many physiological and nonphysiological factors (Farina et al. 2004). A key assumption of this methodology is that neural modularity can be inferred from the common features extracted from muscle activity during movement, irrespective of their source. Prior studies that have found low-dimensional representations of EMG data sets (Ivanenko et al. 2004; Ting and Macpherson 2005) suggest that this assumption may be reasonable. Nonetheless, factorization analysis is not able to dissociate feedforward from feedback contributions to EMGs, nor does it explicitly account for confounds due to muscle dynamics or presuppose functional significance to the modules extracted. Thus if taskspecific sensory feedback or muscle dynamics substantially affect muscles independently, then the ability to extract shared modular building blocks from EMGs would be jeopardized (Steele et al. 2013). Ultimately, more direct measures of neural activation are needed to reinforce or clarify interpretation of results from these EMG-based analyses. In addition, the neurophysiological basis of the control scheme, encompassing how precisely neural outputs are generated and adjusted, is not addressed by this factorization approach.

In this study we considered dimensionality, one aspect of control. Other considerations, such as the flexibility of motor outputs or how a control strategy affects learning (Jacobs and Jordan 1993; Mussa-Ivaldi 1999), may provide additional constraints on what types of control architectures are feasible or useful. Furthermore, our approach does not exhaust the issue of dimensionality. We defined dimensionality in terms of control modules needed for performance of a single motor task, but the total number of modules across all tasks (reflecting the overall parsimony of a strategy) may also be pertinent to control, and is indeed discussed as a trade-off for some architectures. There may also be alternative dimensionality metrics that provide complementary perspectives. For instance, the number of temporal patterns could be assessed as a ratio of time domain (muscle waveform) dimensionality, effectively the minimal set of patterns capable of reconstructing any arbitrary signals of similar bandwidth and duration. Finally, we defined low dimensionality as beneficial; however, there may also be drawbacks to such architectures. The interdependence of muscles in a synergy, for instance, could actually complicate the performance or learning of certain tasks, and perhaps even result in certain combinations of muscle activations being unattainable (McKay and Ting 2008; Tresch and Jarc 2009).

We also recognize the difficult and subjective nature of assessing "good enough" EMG reconstructions. Whenever the number of modules $(k)$ is less than the number of muscle EMGs $(m)$, there will always be a residual error associated with the EMG estimates (Eq. 1). Clearly there is a trade-off between the reduction in dimensionality $(\mathrm{k} / \mathrm{m})$ and the ability to account for the data $\left(R^{2}, \mathrm{VAF}\right)$. Therefore, it is not surprising that more stringent evaluation criteria necessitate more modules to reconstruct the EMGs. Likewise, it is not surprising that a lower number of modules can be said to account for muscle EMGs if the evaluation criterion is more lenient. This reflects the classic trade-off between type I and type II errors when setting a threshold. Thus evaluation criteria must be carefully selected and assessed to avoid experimenter bias and results that are inextricably linked to specific methodological choices. To address these limitations, we checked the sensitivity of our methods by varying our IndividualMuscle reconstruction criterion from $R^{2} \geq 0.8$ to $R^{2} \geq 0.7$ and the Active muscle threshold from $20 \%$ to $10 \%$. We found that within a reasonable range these parameters had little effect on our qualitative findings. Nevertheless, the impact of imprecise EMG reconstruction on functional performance remains unclear because of the highly nonlinear mapping from muscle activity to movement dynamics. On one hand, even small errors in muscle activity could result in dramatic consequences in task performance (Alessandro et al. 2013; de Rugy et al. 2013). On the other hand, the possibility exists that a fairly crude approximation of EMG activity may be sufficient to provide basic functionality (Kargo et al. 2010). 
There are also trade-offs to methodological choices made for extracting modules. For instance, we chose to extract modules from stride-averaged EMG data, similar to many previous studies on modularity (d'Avella et al. 2013; Cappellini et al. 2006; Ivanenko et al. 2004; Overduin et al. 2008, 2012; Torres-Oviedo et al. 2006). The benefit is that the averaging process acts as a low-pass filter to remove stride-specific noise and transient artifacts in EMG. The trade-off is that potentially relevant stride-to-stride variability in muscle activations is also reduced (see Table 1). Extracting modules from individual stride EMGs could overcome this this limitation; however, studies that have used this approach have also needed to use simplifying assumptions that affect variance, such as condensing the EMG patterns into down-sampled time bins (Chvatal and Ting 2012; Safavynia and Ting 2012). Furthermore, the latter approach would require us to introduce an additional analysis step to condense the extracted modules from each stride into the single consolidated set of shared modules used by the neural controller. When we did experiment with extracting synergies from individual strides we found, as expected, that they were similar to those extracted from stride-averaged EMG. Also as predicted, when EMGs were reconstructed for individual strides the quality of reconstruction was slightly degraded. For instance, when five modules were extracted from forward walking EMGs, the Grouped-Muscle $R^{2}$ decreased from $0.94 \pm 0.02$ to $0.87 \pm$ 0.04. However, since individual stride EMGs exhibit both physiolog- ically relevant and irrelevant variability (e.g., due to measurement noise), it is only possible to speculate about the source and significance of this difference.

\section{RESULTS}

\section{$E M G$}

EMGs were comparable to prior literature. Average EMG envelopes (Fig. 2) appeared qualitatively similar to published recordings of forward walking (Ivanenko et al. 2004; Winter and Yack 1987), backward walking (Cappellini et al. 2010; Ivanenko et al. 2008), and stepping in place (Grasso et al. 2004). We are unaware of published EMGs for sideways walking. Our foot muscle recordings also appear qualitatively consistent with published data (Courtine et al. 2007; Gray and Basmajian 1968; Perry 1992; Reeser et al. 1983; Saraswat et al. 2010).

\section{Task-Dependent Architectures}

Task-dependent patterns and synergies were qualitatively similar to those found in prior studies (Fig. 3). However,

Table 1. Variance of normalized EMG signals from entire 30-s muscle recordings and from stride-averaged waveforms used in factorization analysis

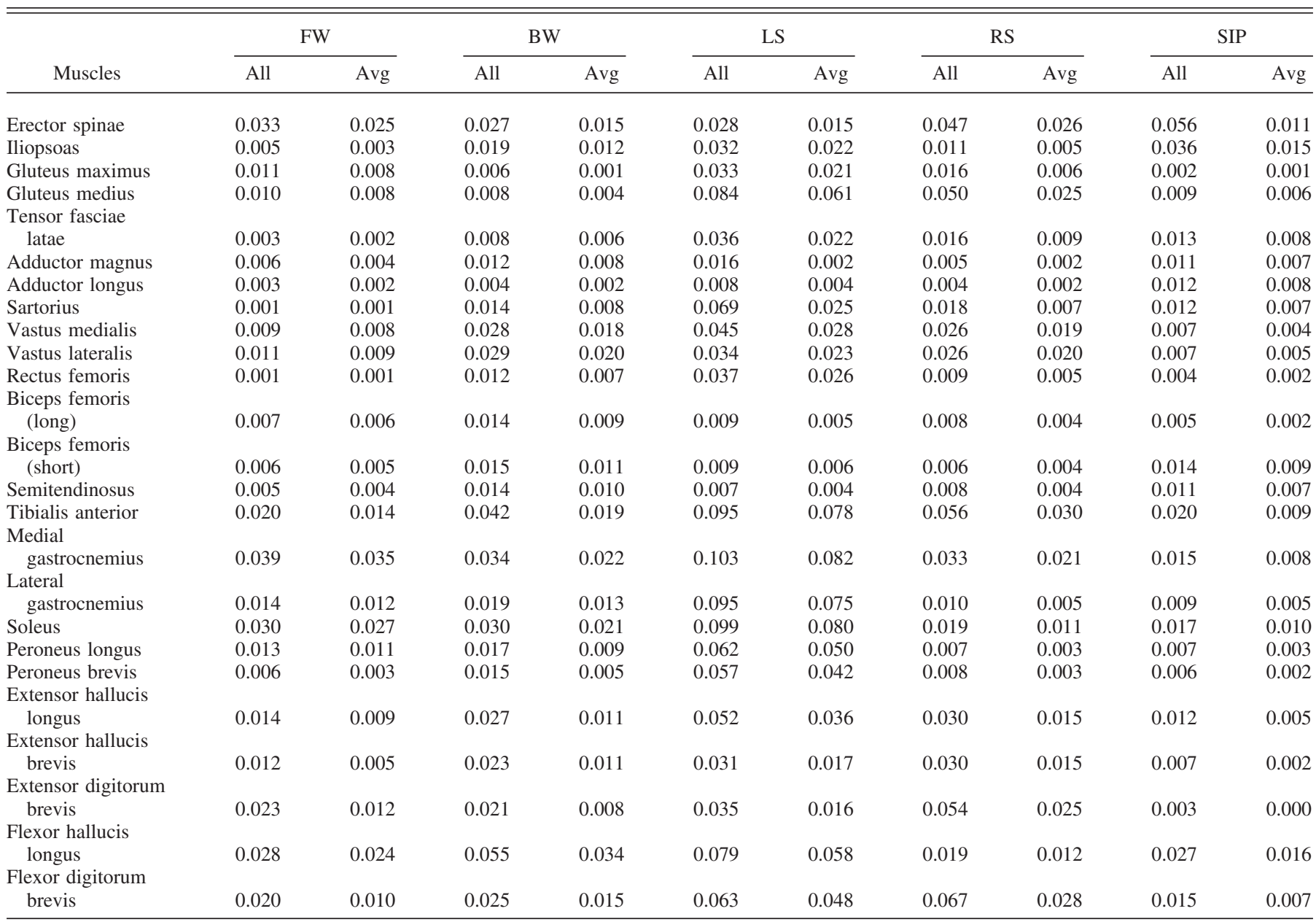

Subject-averaged means are reported for variance of normalized EMG signals from entire 30-s muscle recordings (All) and from the stride-averaged waveforms (Avg) used in factorization analysis. FW, forward walking; BW, backward walking; LS, sideways walking to the left; RS, sideways walking to the right; SIP, stepping in place. 
Fig. 2. Task-specific EMGs. Average EMGs for the right leg are depicted for each task along with standard deviation across subjects $(n=8)$. EMGs are plotted across a gait cycle from 0 to $100 \%$, with 0 corresponding roughly to right foot strike (see METHODS for details). EMGs are plotted in normalized units as a fraction of maximum activation magnitude. Average stance durations were similar between tasks $(58-62 \%)$ and are shown for reference.

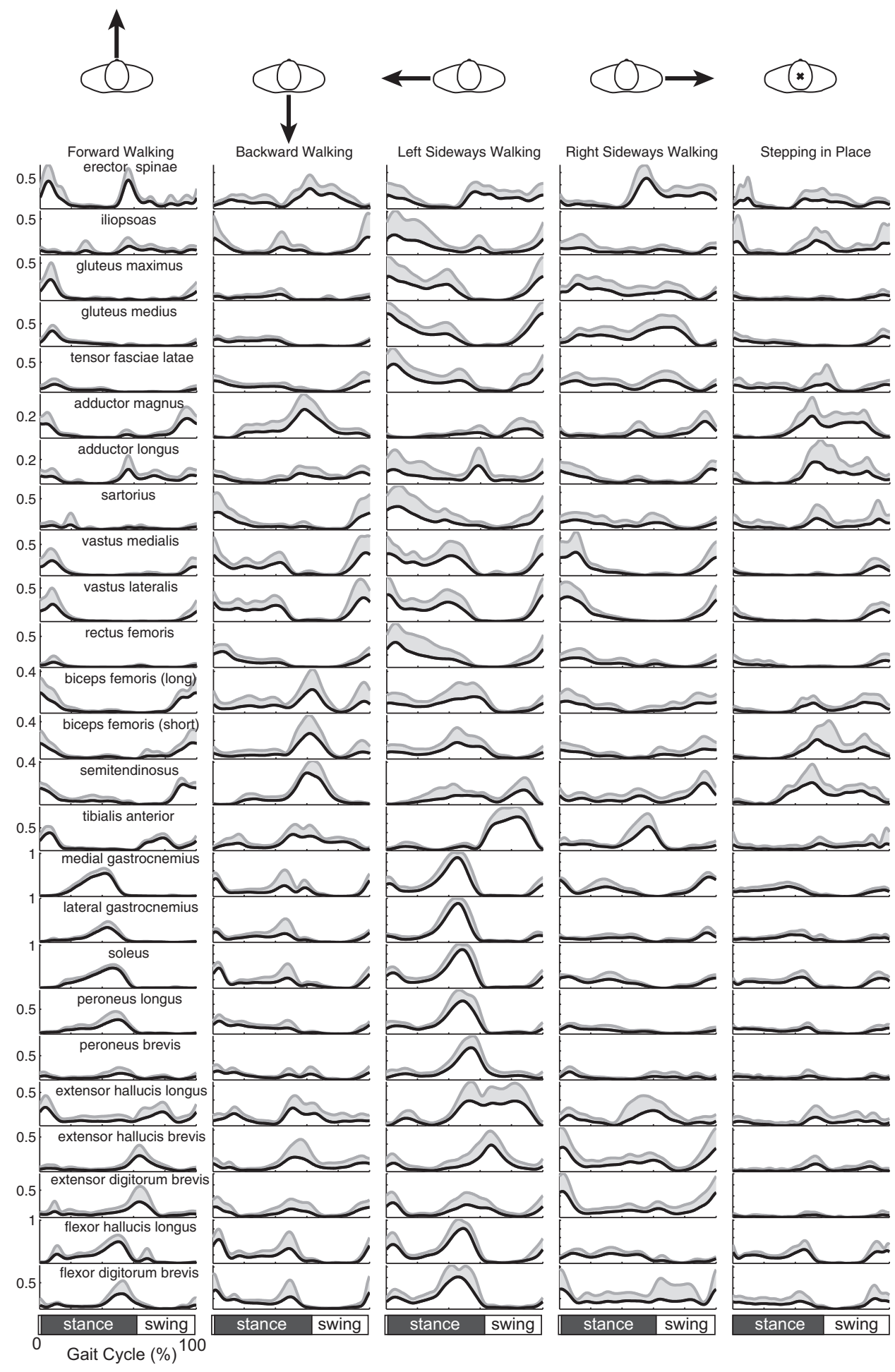

previous studies (Cappellini et al. 2006; Ivanenko et al. 2006). An additional pattern (4) reflects the activation of intrinsic foot muscles that have not traditionally been recorded in studies of modular control.

A task-dependent architecture was found to reduce control dimensionality by roughly $65 \%$ compared with controlling each muscle individually. A relatively low number of taskdependent modules were sufficient to reconstruct EMGs for each task. On average $5.8 \pm 0.7$ modules (Fig. 4) reconstructed patterns $(2,3$, and 5$)$ from midstance to midswing, similar to 


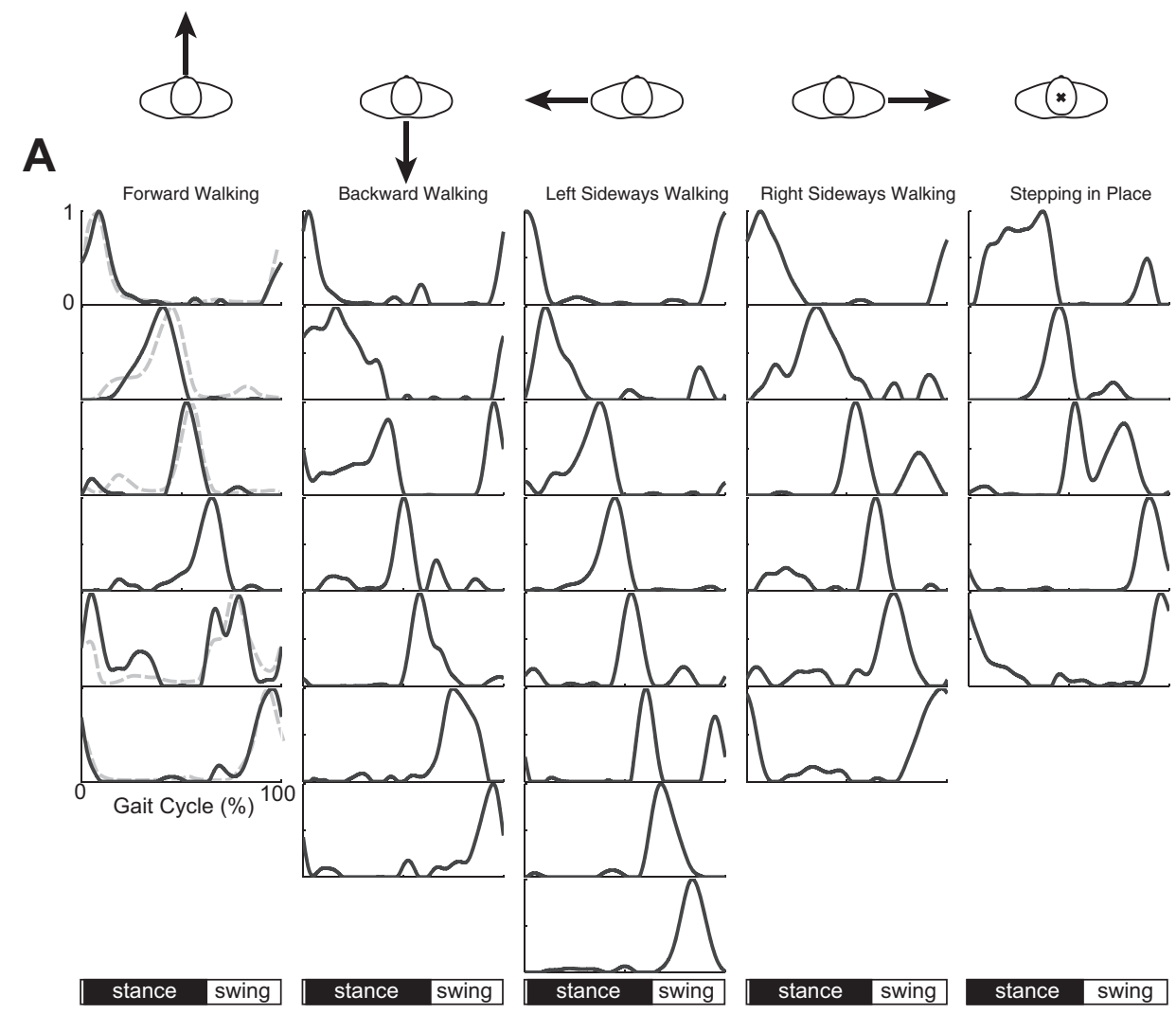

B

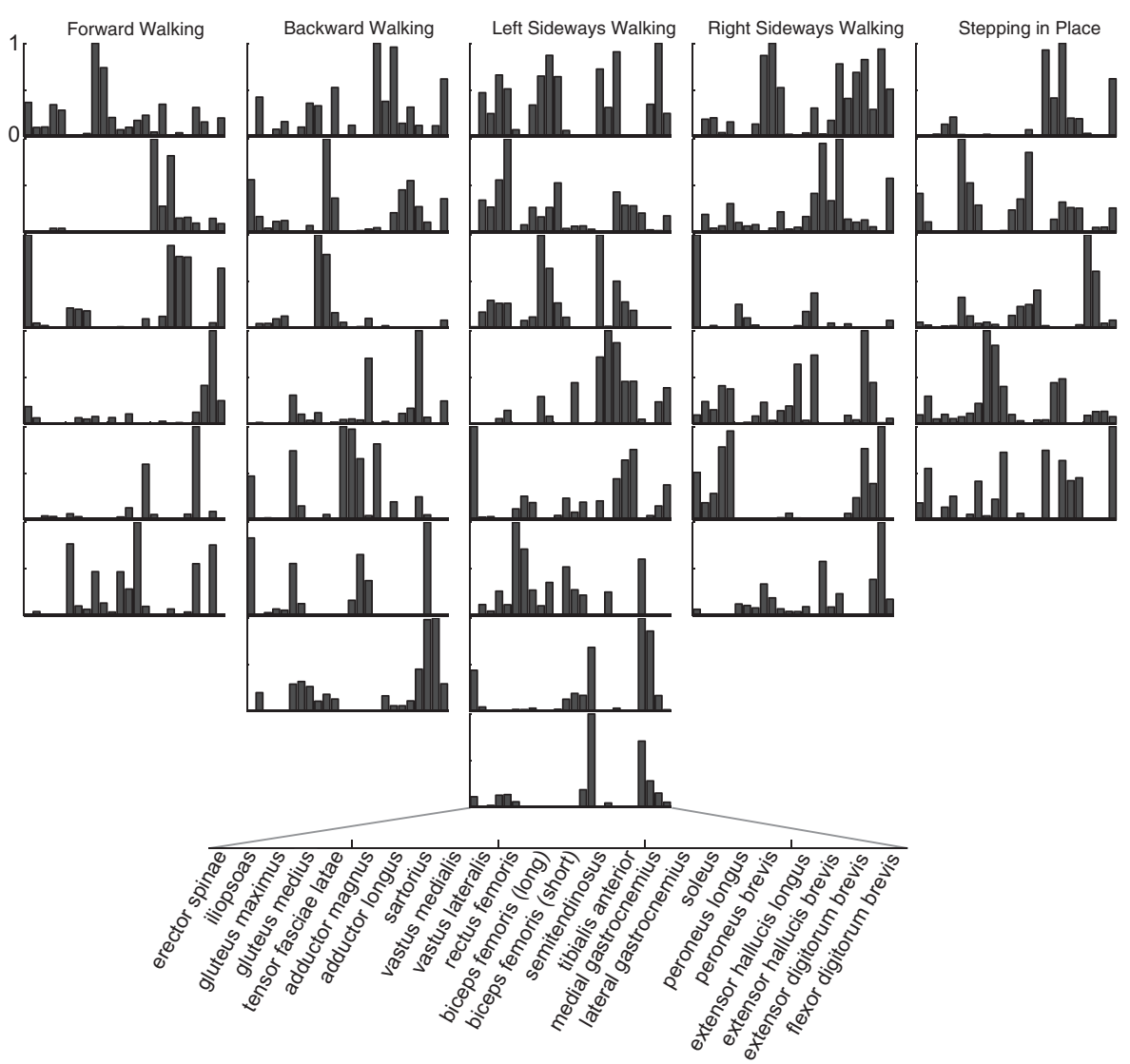

Fig. 3. Representative task-dependent patterns and synergies. Temporal patterns $(A)$ and the corresponding muscle synergies $(B)$ are shown for a representative subject for each task. For visualization purposes each pattern and synergy is normalized to unity. Average stance and swing durations are depicted for reference. In $A$, 5 of the 6 forward walking patterns were in strong agreement with patterns from prior literature (gray dashed lines; Ivanenko et al. 2006). The remaining pattern (4) reflects activation of intrinsic foot muscles, which were not recorded in previous studies. 
A

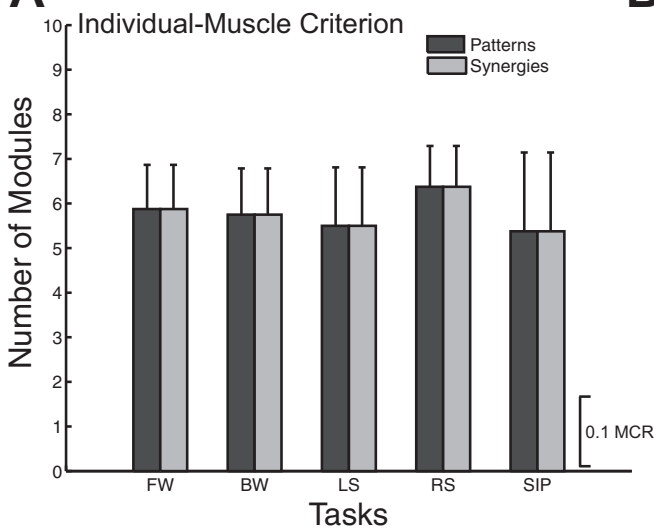

B

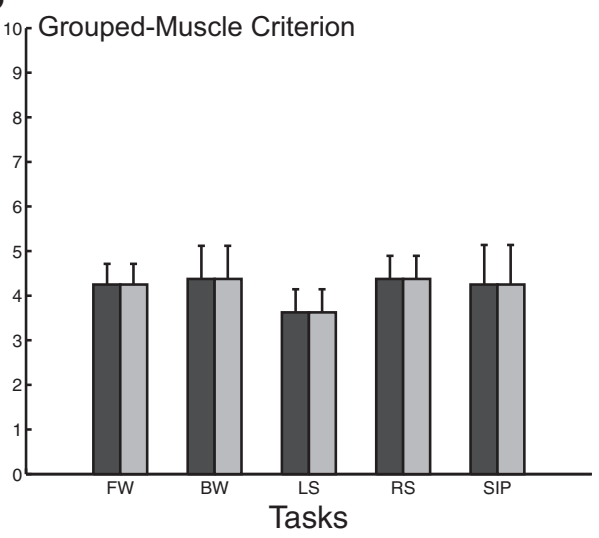

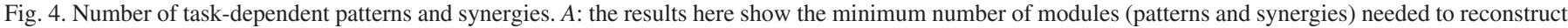

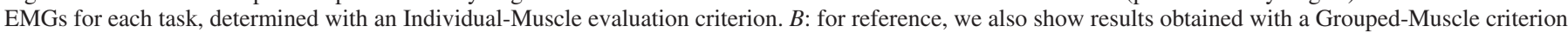

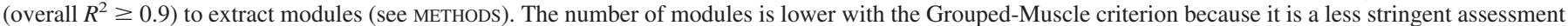

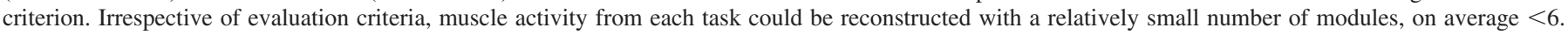

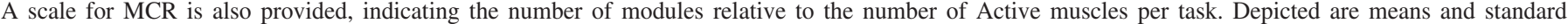
deviations across subjects $(n=8)$.

$>90 \%$ of the Active muscles (15.4 \pm 2.8$)$ for each task, yielding an $\mathrm{MCR}=0.38$. The alternative estimate, $\mathrm{MCR}_{\mathrm{A}}$, yielded a similar value of $\sim 0.35$ (Fig. 5). This was largely independent of the number of control modules, although roughly 7 modules were needed in order to reconstruct $90 \%$ of all muscles recorded.

The total number of task-specific modules scaled roughly linearly with the number of tasks. This qualitative trend was
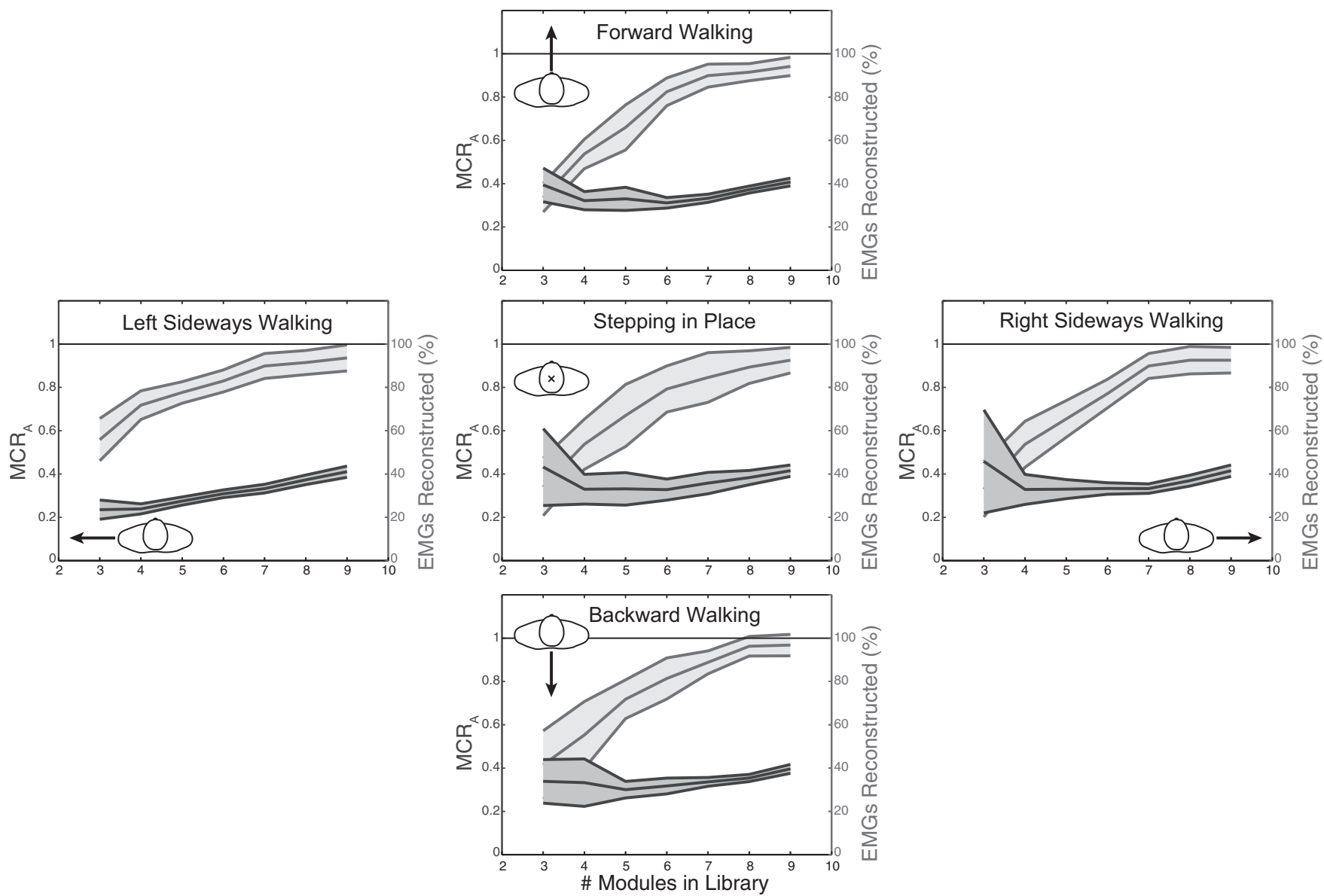

Fig. 5. Alternate modular control ratio for task-dependent architecture. The alternate modular control ratio $\left(\mathrm{MCR}_{\mathrm{A}}\right.$; black line, dark shading) and the percentage of EMG waveforms that were well-reconstructed (gray line, light shading) are plotted for each task as a function of the library size (i.e., the number of neural control modules). $\mathrm{MCR}_{\mathrm{A}}$ is defined as the number of modules in the library divided by the number of muscles being controlled, which we approximated as the number of muscles whose EMGs were well-reconstructed $\left(R^{2} \geq 0.8\right)$. With task-dependent modules the $\mathrm{MCR}_{\mathrm{A}}$ was found to be roughly $0.3-0.4$, when reconstructing $>90 \%$ of the muscles. Depicted are means and standard deviations across subjects $(n=8)$. 
independent of the precise criterion used to select the number of modules. With the Individual-Muscle criterion, a total of $28.9 \pm 3.4$ modules were extracted across tasks (i.e., the sum of the 5 task-dependent libraries).

\section{Task-Independent Architectures}

The number of modules in the shared library increased with the number of locomotor tasks analyzed (Fig. 6A). The size of the shared synergy library increased from $5.8 \pm 1.0$, when only extracted from forward walking, to $14.5 \pm 1.9$, when extracted from all tasks. Thus the size of the shared synergy library was roughly equivalent to the number of Active muscles per task (15.4). The size of the shared pattern library also increased with tasks but leveled off at $9.9 \pm 1.1$ patterns (Fig. 6A). When modules were extracted from three or more tasks the number of shared synergies needed was significantly higher than the number of shared patterns needed to reconstruct EMGs $(P<$ 0.036).

An architecture using task-independent patterns was found to reduce dimensionality by $45 \%$. A subset of the shared pattern library was required to reconstruct EMGs for each task. On average, $8.3 \pm 0.2$ of the shared patterns were needed to control the roughly 15 Active muscles per task (Fig. $6 B$ ). The MCR of 0.54 was slightly higher than the $\mathrm{MCR}_{\mathrm{A}}$ estimate of 0.41 , given reconstruction of $90 \%$ of all EMGs (Fig. 7A). MCR $\mathrm{A}_{\mathrm{A}}$ was found to decrease when more muscles were analyzed (Fig. 7), for instance, from 0.68 to 0.41 when computed from 12 vs. 25 muscles. It is also worth noting one qualitative observation, namely, that the library of shared patterns tended to look like a sequence of roughly equidistant impulses (Fig. 8A).

An architecture using task-independent synergies was found to reduce dimensionality during each task by $\sim 35 \%$. We found that not all synergies in the shared library needed to be recruited for each task. A subset of the modular library, $9.7 \pm$ 1.1 of the shared synergies (Fig. $6 B$ ), were required to control $\sim 15$ Active muscles per task, yielding an $\mathrm{MCR}=0.63$. This was in agreement with the alternate estimate $\mathrm{MCR}_{\mathrm{A}}=0.65$ when $80 \%$ of all muscles were well-reconstructed (Fig. $7 A$ ). This $80 \%$ threshold was selected here because, on average, even a library of 19 shared synergies was unable to reconstruct $90 \%$ of muscle EMGs. When computed from the 20 nonfoot muscles and 12 commonly recorded muscles, $\mathrm{MCR}_{\mathrm{A}}$ was found to be similar, 0.64 and 0.71 , respectively (Fig. 7, $B$ and $C$ ). Because the number of synergies in the shared library was still increasing when the fifth task was added (Fig. 6A), and a large subset of the library was consistently needed for each task, it is likely that the MCR estimates presented here are underestimates. Nevertheless, a few of the shared synergies did suggest functional significance, the coupled activation of muscles that perform similar biomechanical function (e.g., adductor magnus and longus, soleus and peroneus muscles; Fig. 8B). However, many "single-muscle" synergies were also found within the shared library (Fig. 8B). Typically more than onethird of the extracted synergies were dominated by a single muscle weighting.

Neither patterns nor synergies extracted from forward walking were able to satisfactorily reconstruct EMGs during other modes of locomotion. When applied to the other four tasks, the forward walking patterns yielded an overall Grouped-Muscle $R^{2}$ of $0.75 \pm 0.05$. On average, only $28 \pm 12 \%$ of the individual EMGs were well-reconstructed $\left(R^{2} \geq 0.8\right)$. Allowing patterns to shift together by up to $10 \%$ of the gait cycle improved reconstructions slightly, increasing Grouped-Muscle $R^{2}$ by $\sim 0.04$ to $0.79 \pm 0.05$. EMG reconstructions from forward walking synergies resulted in a lower Grouped-Muscle $R^{2}$ of $0.61 \pm 0.12$, and only $22 \pm 9 \%$ of the individual EMGs were well-reconstructed.

Extracting shared modules from unidirectional locomotion (self-selected cadence, fast cadence, slow cadence, tiptoe, and $20 \%$-incline walk) yielded results similar to those found during multidirectional locomotion (Fig. 9). For example, we found that $9.3 \pm 0.9$ shared synergies and $8.4 \pm 0.7$ shared patterns were needed to reconstruct EMGs for each variation of forward walking (compared to 9.7 synergies and 8.3 patterns for each multidirectional task). Again, we found that roughly one-third of the shared synergies appeared to be "single-muscle" synergies. The average MCRs of 0.53 for shared synergies and 0.48 for shared patterns were slightly lower than the 0.63 and 0.54 estimates from multidirectional locomotion.

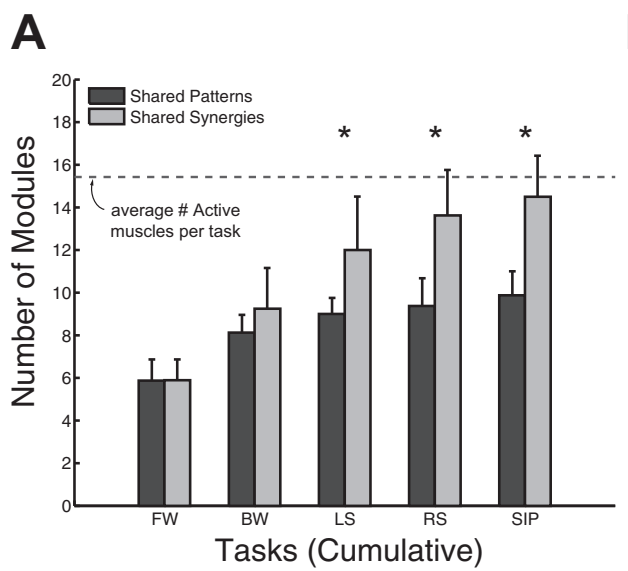

B

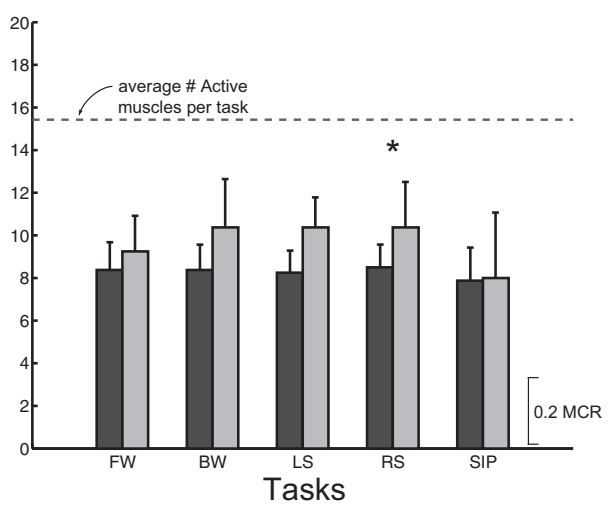

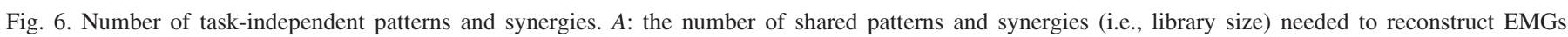

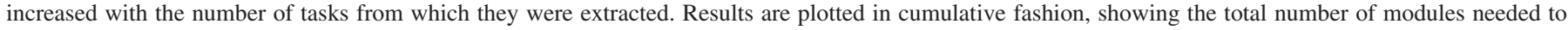

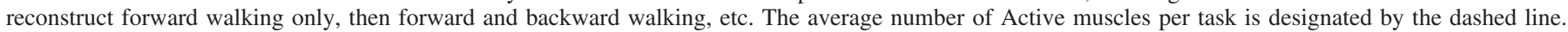

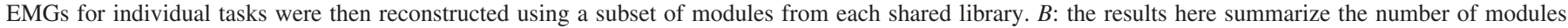

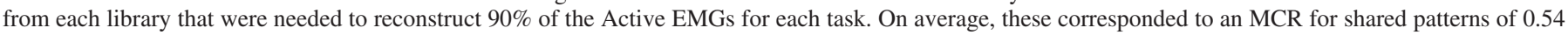
and for shared synergies of 0.63 . *Statistical significance $(P<0.05)$ between the number of shared synergies vs. patterns. 
Fig. 7. $\mathrm{MCR}_{\mathrm{A}}$ for task-independent architectures. $\mathrm{MCR}_{\mathrm{A}}$ (black line, dark shading) and the percentage of EMG signals that were well-reconstructed (gray line, light shading) are plotted as a function of the number of shared control modules. MCR $\mathrm{A}_{\mathrm{A}}$ is defined as the number of modules recruited to perform a task divided by the number of muscles being controlled, approximated as the number of well-reconstructed EMG waveforms $\left(R^{2} \geq\right.$ $0.8)$. Left: results for a control strategy using a shared library of fixed neural patterns as its building blocks. Right: results for a strategy using a shared library of fixed muscle synergies. Results were computed using all 25 of the EMGs recorded $(A)$, all 20 of the nonfoot muscle EMGs $(B)$, and EMGs from 12 commonly recorded leg muscles $(C)$ (see METHODS for details). Depicted are means and standard deviations across subjects $(n=8)$.
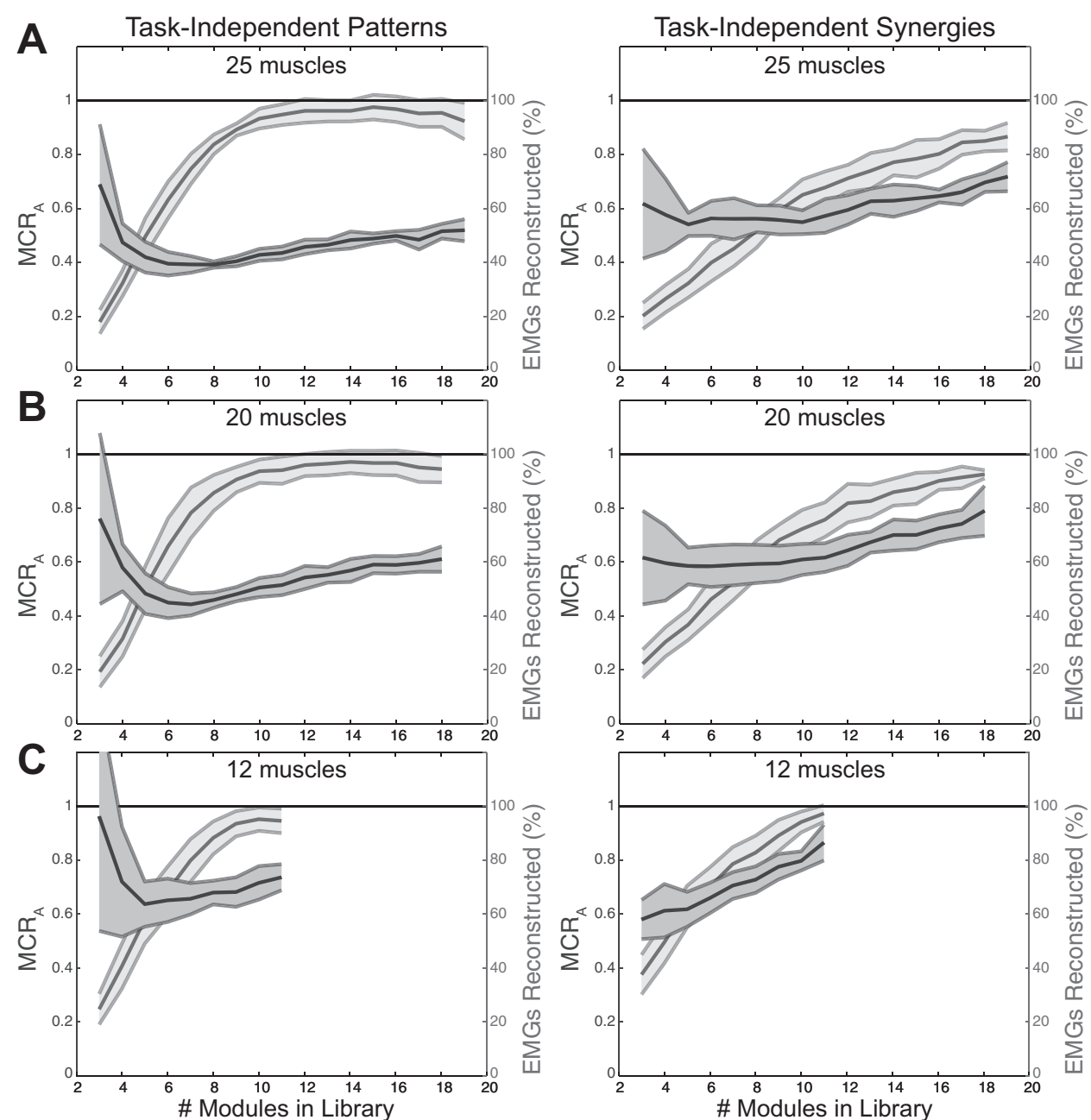

\section{DISCUSSION}

We found that while simple modular architectures could theoretically reduce dimensionality compared with independent muscle control, controllers relying on shared neural primitives may be limited in their ability to coordinate muscle activity for various tasks. The efficacy of shared primitives may thus depend on how they can be adapted by or embedded within the sensorimotor control system. The results obtained here based on simple neural architectures highlight the need for more sophisticated formulations of modular control or alternative motor control hypotheses and motivate future research to identify specific, testable neural mechanisms that can accommodate muscle coordination for disparate locomotor tasks.

\section{Simple Modular Architectures}

Primitives extracted from individual tasks were found to reduce dimensionality substantially (Fig. 4, Fig. 5), indicating the potential utility of neural modularity. However, this taskspecific architecture represents a best-case scenario for reducing dimensionality with fixed modules, and there are trade-offs to this specificity. For instance, if these task-specific modules were interpreted in the strictest and simplest sense, then they would be indistinguishable from strategies involving stored motor programs, requiring an ever-increasing number of modules to perform additional tasks. Thus they would have little predictive value as a theory of motor control (de Rugy et al. 2013).

Various interpretations of task-specific modules might address this issue. First, each library of modules might support a limited subset of behaviors, perhaps different from those tested in this study. For example, analysis of eight muscle EMGs suggested that modules might be used for a range of levelground, forward walking speeds (Clark et al. 2010). Second, some literature has suggested that an overcomplete representation of neural control may actually be an efficient strategy for motor learning (Flanders 2011; Lewicki and Sejnowski 2000), which could potentially constitute a different benefit. This would perhaps be consistent with previous observations that the number of modules extracted from muscle EMGs during locomotion increases throughout adolescent development in several animal species including the human (Dominici et al. 2011). However, the overcomplete representation might also suggest that muscle activity is the result of synergies that are dynamically constructed (and then deconstructed) by the nervous system, rather than neural primitives that are stored and then recruited (Llinás and Roy 2009). This interpretation would differ from the hypothesis that a finite number of building blocks might be flexibly recombined to generate muscle activations for disparate motor behaviors. Third, the apparent need for an ever-increasing number of task-specific 
A

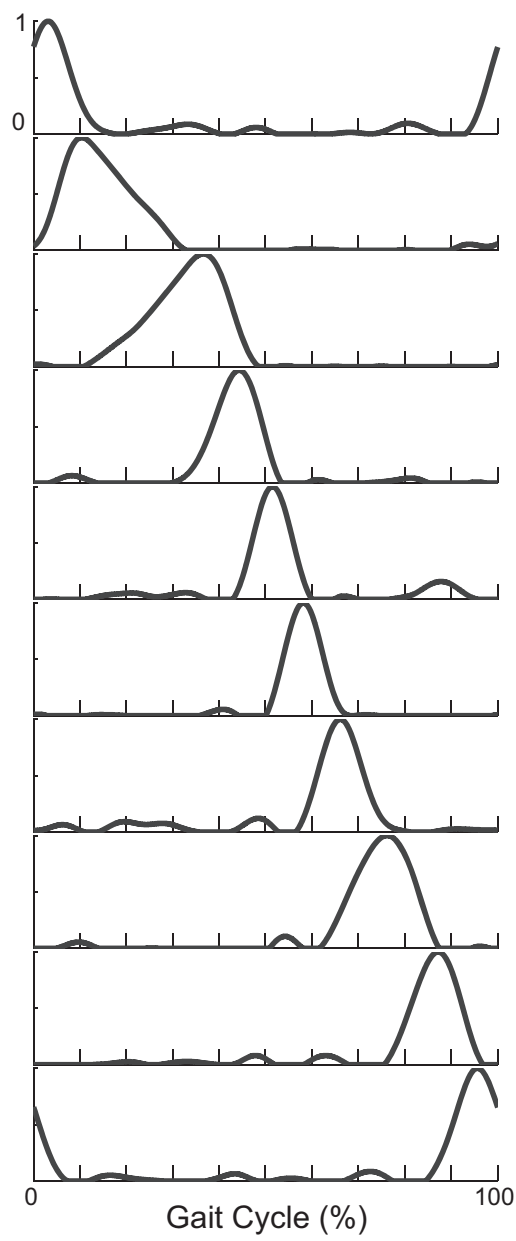

B

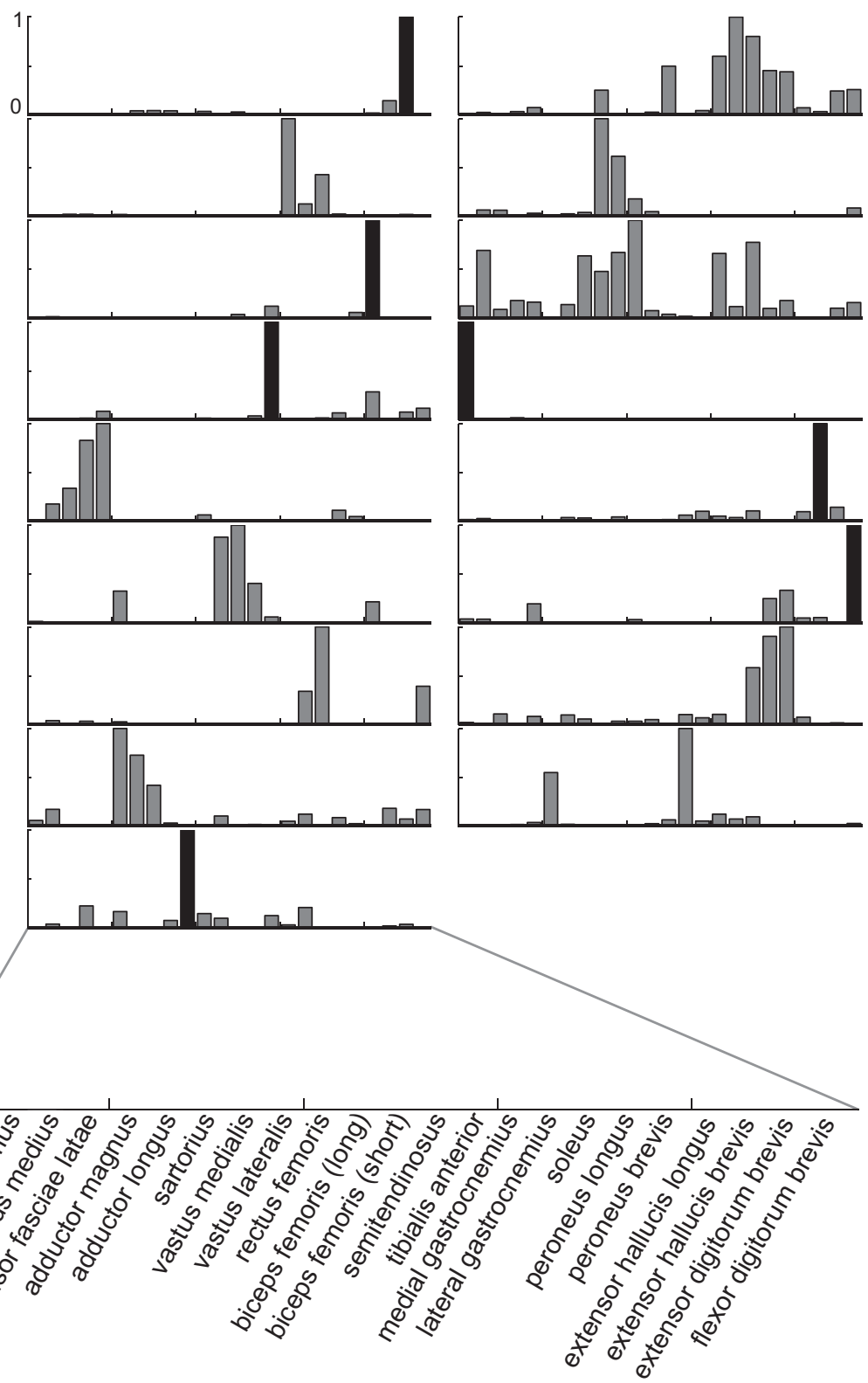

Fig. 8. Representative task-independent patterns and synergies. Shared patterns $(A)$ and shared synergies $(B)$ are shown for a representative subject (same as Fig. 3 ). These depict the minimum set of shared patterns (10) and the minimum set of shared synergies (17) needed to reconstruct activity from this subject's 24 EMGs, recorded during the 5 multidirectional tasks. For visualization purposes each pattern and synergy is normalized to unity. In $B$, over one-third of the synergies exhibited a dominant single-muscle weighting (shown as a darkened bar).

modules might simply be a result of limitations in our extraction methodology. These task-dependent modules might instead represent shared primitives that are adapted by sensory feedback (Cheung et al. 2005; Lacquaniti et al. 2012; Ting 2007) and are thus difficult to discern with conventional statistical analyses, which describe commonalities in EMG due to both feedforward and feedback contributions. While afferent feedback is known to contribute to neural activity during locomotion (Duysens et al. 1998; Grey et al. 2001; Sinkjær et al. 2000;), few studies have tried to account for this feedback in the extraction of neural primitives (but see Kargo and Giszter 2008). Further study is required to evaluate these possibilities, in particular studies that can discriminate modularity adapted by sensory feedback from other possible mechanisms.

When shared temporal patterns were extracted they were found to reduce dimensionality (Fig. 6, Fig. 7) but nonetheless may have practical limitations for controlling disparate motor behaviors. The shared library was relatively small ( $\sim 10$ modules), suggesting a parsimonious solution to motor control. However, the pattern library itself appeared to be a sequence of time-shifted impulses (Fig. 3A), similar to that found in a previous study (Safavynia and Ting 2012). This perhaps reflects rhythmic generators in the nervous system (Giszter et al. 

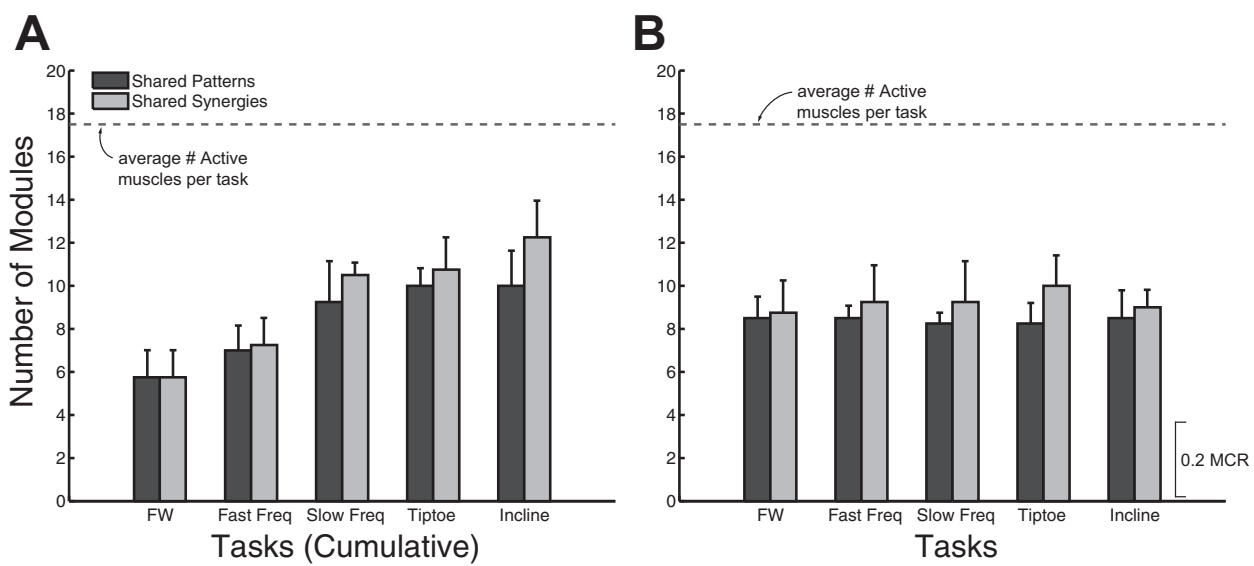

Fig. 9. Number of task-independent patterns and synergies for unidirectional locomotion. In $A$ the size of each shared library is shown. Similar to multidirectional locomotion (Fig. 6), the number of modules increased with the number of tasks from which they were extracted. Results are again plotted in cumulative fashion, showing first the number of modules needed to reconstruct self-selected forward walking only (FW), then progressing incrementally to include each of the other tasks: fast step frequency walking (Fast Freq), slow step frequency walking (Slow Freq), tiptoe walking (Tiptoe), and walking up an incline (Incline). In $B$ the results show the minimum subset of the shared modules needed to reconstruct $90 \%$ of the Active EMGs for each task. On average, the MCRs for shared patterns and synergies during unidirectional locomotion were 0.48 and 0.53 , respectively. Depicted are means and standard deviations across the subset of subjects $(n=4)$ who performed these unidirectional locomotor tasks.

2007; Ivanenko et al. 2004; McCrea and Rybak 2008; Patla et al. 1985; Zehr 2005). Alternatively, this solution may simply reflect mathematical convergence of the pattern library toward maximum temporal dimensionality, the set of temporal basis functions capable of reconstructing any arbitrary signals of similar filtering and duration. This brings into question the usefulness of this strategy for dimensionality reduction because tasks involving higher-frequency muscle contractions or cycle durations longer than those tested $(\sim 0.9-1.2 \mathrm{~s})$ would require substantially more patterns.

Shared muscle synergies also provided some reduction in dimensionality but exhibited characteristics that may be undesirable as a basis for controlling various motor behaviors. For instance, we found that the number of shared synergies needed was roughly as large as the number of muscles controlled (Fig. $6 A$ ). Also, the modest reduction in dimensionality was likely an overestimate of the benefit, as it was only applicable to a subset of the 25 muscles recorded (roughly 15-19 based on estimates of MCR). Furthermore, we found that many synergies were characterized by a dominant weighting (Fig. 8B), suggesting individual muscle control. We found similar results when considering a set of unidirectional locomotor tasks, which also required a relatively high number of synergies to reconstruct EMGs (Fig. 9B). We did observe a mild improvement in MCR (0.53 unidirectional vs. 0.63 multidirectional); however, this may simply be due to anatomically related muscles (e.g., gastrocnemius and soleus) that function similarly during forward-direction gait but whose activations are known to dissociate during other tasks such as curvilinear walking (Courtine et al. 2006) and cycling (Wakeling and Horn 2009). Thus our findings appear consistent with previous studies that have questioned the compatibility or utility of muscle synergies for explaining empirical data (Flanders 2011; Giszter et al. 2007; Kutch et al. 2008; Macpherson 1991; de Rugy et al. 2013; Soechting and Lacquaniti 1989; Wakeling and Horn 2009) and contrary to the hypothesis that fixed synergies are the building blocks of movement. A full discussion of the competing evidence is beyond the scope of this article but is summarized in various reviews (e.g., Alessandro et al. 2013; Tresch and Jarc 2009).

\section{Beyond Simple Modular Architectures}

While the simple control architectures tested in this study may be considered naive or oversimplified models of modularity, they bring to bear several important questions. In particular, if these architectures are too simplistic, then what specific modular extensions or motor control alternatives are proposed? The principal benefit of these simple models is that they are well-defined, testable, and computationally tractable. While the inclusion of additional complexity (e.g., sensory feedback) may seem obvious and necessary, the precise implementation within a modular control architecture remains nontrivial in the absence of well-understood neural mechanisms underlying sensorimotor integration (Giszter et al. 2007; Tresch and Jarc 2009). Thus the scientific challenge remains: can more sophisticated models be formulated in ways that are specific and falsifiable? Some such alternatives have already been proposed (with varying levels of specificity), and below we discuss our findings in the context of these hypothesized neural architectures.

Modular architectures could potentially simplify locomotor control further and address limitations observed with shared primitives but may require more complex organizations or building blocks. For example, an alternative modular strategy might involve a "mixed" architecture, comprised of both shared and task-specific modules. For instance, d'Avella and Bizzi (2005) found that muscle activations in various types of frog locomotion (walking, swimming, jumping) could be reproduced using mostly shared synergies and a few task-dependent synergies. In contrast, Chhabra and Jacobs (2006) found that human reaching could be explained by principally taskdependent synergies, with a small number of shared ones. Other plausible architectures might use time-varying synergies (d'Avella et al. 2003), multilevel hierarchical structures (Giszter et al. 2007; McCrea and Rybak 2007, 2008; Tessitore et al. 2013), modularity within neuronal networks (Grillner 2011; Hart and Giszter 2010; Stein 2008), sensory feedback adapta- 
tion (Cheung et al. 2005; Duysens et al. 1998, 2013; McCrea and Rybak 2008), or perhaps muscle synergies that are modifiable within a limited vector space rather than fixed (Drew et al. 2008). However, it is worth noting that some of these possibilities begin to touch upon the difficult practical question of what actually constitutes neural or biological modularity (Lacquaniti et al. 2013).

Although explicitly testing a "mixed" modular strategy was outside the scope of our study, the $\mathrm{MCR}_{\mathrm{A}}$ metric provides one way to assess such architectures. Since $\mathrm{MCR}_{\mathrm{A}}$ is computed over a wide range of library sizes, we can evaluate the consequences of using a smaller number of shared modules (i.e., not capable of reconstructing the majority of muscle activations). For synergies we observed roughly constant $\mathrm{MCR}_{\mathrm{A}}(\sim 0.6$; Fig. $6 A)$. Thus, for instance, a library of 6 shared synergies could theoretically control 10 muscles, leaving the remaining 15 muscles recorded in this study to be controlled by task-specific modules or by other nonmodular neural processes. $\mathrm{MCR}_{\mathrm{A}}$ for shared patterns was also roughly constant, 0.4 for all library sizes except the very smallest $(<5)$. Thus "mixed" architectures may offer limited benefits in reducing control dimensionality for a subset of muscles but would also exhibit drawbacks associated with a task-specific organization. Nevertheless, further investigation is required to understand the full implications of "mixed" architectures, as well as to address technical challenges related to extracting shared vs. taskspecific primitives.

The results presented here might also be interpreted as consistent with other neuromotor control theories. For instance, some researchers have suggested that task-specific modules may be a by-product of biomechanical constraints (Kutch and Valero-Cuevas 2012) or regularities in learned muscle patterns (de Rugy et al. 2012) or emerge from optimal control theory (Chhabra and Jacobs 2006; de Rugy et al. 2013; Todorov 2004) rather than reflect tangible modules employed by the neural controller. Others have interpreted the extracted primitives as indications of a distributed motor control organization, noting that even higherorder primitives that account for increasingly smaller amounts of variance in data reconstruction may still represent important features of the behavior (Flanders 2011; Santello et al. 1998). Thus a small number of shared synergies might be employed broadly, while additional task-specific mechanisms (perhaps modular or not) would provide finer motor control. This bears some similarities to the "mixed" modular strategy discussed above, which would be predicted to have limited benefits for reducing dimensionality when using a small number of shared primitives. However, it is also possible that the principal benefit of modularity or other neural control strategies is actually unrelated to dimensionality reduction during performance of a task. For instance, modularity might be more pertinent to the initial learning of a motor task (Berger et al. 2013; Bernstein 1967; Dominici et al. 2011).

\section{Further Methodological Considerations}

Various methodological considerations are detailed in Assumptions and Limitations above. There we address limitations related to the inference of neural control strategies from EMG signals, the measurement and use of dimensionality as an indicator of motor control complexity, the definition of EMG reconstruction criteria, and the extraction of neural primitives from stride-averaged EMG data. For brevity, we do not rediscuss these topics here but rather address further methodological considerations related to the interpretation of results.

It is worth noting that our results and conclusions may differ from some prior experimental studies using similar EMG decomposition methods. Here we speculate as to methodological reasons why some previous findings may have been interpreted in stronger support of simple shared building blocks that can reduce dimensionality and accommodate various motor tasks. First, we used a more stringent EMG reconstruction criterion to identify the number of neural primitives required. This is because we discovered that conventional GroupedMuscle criteria fail to reconstruct many EMGs (e.g., $40 \%$ of EMGs during forward walking), which likely results in substantial errors in kinetics (Alessandro et al. 2013; de Rugy et al. 2013). Thus, for example, 5 synergies that were conventionally believed to reconstruct 12 EMGs may only reproduce about 7 of them well. Therefore, we introduced the MCR, the ratio of neural control modules to muscles, to help discern practical implications of dimensionality. We also found that similar issues exist if the Individual-Muscle criterion is too lenient, in which case critical features of EMG may be poorly reconstructed or completely missing (Fig. 10A). The use of less stringent Individual-Muscle criteria (e.g., $R^{2} \geq 0.6$ or VAF $\geq$ $75 \%$ ) may underlie more favorable interpretations of shared primitives for explaining empirical muscle activity. Choosing a "good enough" threshold for EMG reconstructions is admittedly a difficult and subjective decision (as detailed in METHODS). Thus Fig. 10A is principally presented to facilitate the reader in forming his/her own opinion as to what constitutes "good enough," and to make transparent the challenge of selecting evaluation criteria.

Our results may also differ from previous studies because of the techniques used for identifying shared modules. Post hoc statistical techniques, such as Monte Carlo simulation or Pearson correlation, have commonly been employed to identify synergies that appear similar. These techniques compare modules that are extracted separately from different tasks or subjects. While these may be useful techniques for comparing similarities between subjects, we found that they may be misleading for intrasubject analysis. For a single subject, a shared neural primitive is defined as one that is used to generate muscle activity for multiple tasks. In this study we enforce this definition by extracting modules from combined sets of EMG data (from all tasks). However, a statistical comparison of modules that are extracted independently can circumvent this definition and can result in mildly correlated modules being statistically defined as identical. For instance, if we applied this statistical comparison to a pair of moderately correlated synergies $\left(R^{2}=0.27\right.$; Fig. $\left.10 B\right)$ from two separate tasks, then we would conclude that these synergies were the same and thus shared (based on critical values for Pearson correlation coefficient with significance level of 0.01). However, this contradicts the qualitative, intuitive assessment that these synergies appear to be quite different. Furthermore, even if there were a method to resolve the quantitative differences (e.g., by computing an average) and to create a shared library of synergies, then it would degrade their ability to generate the 

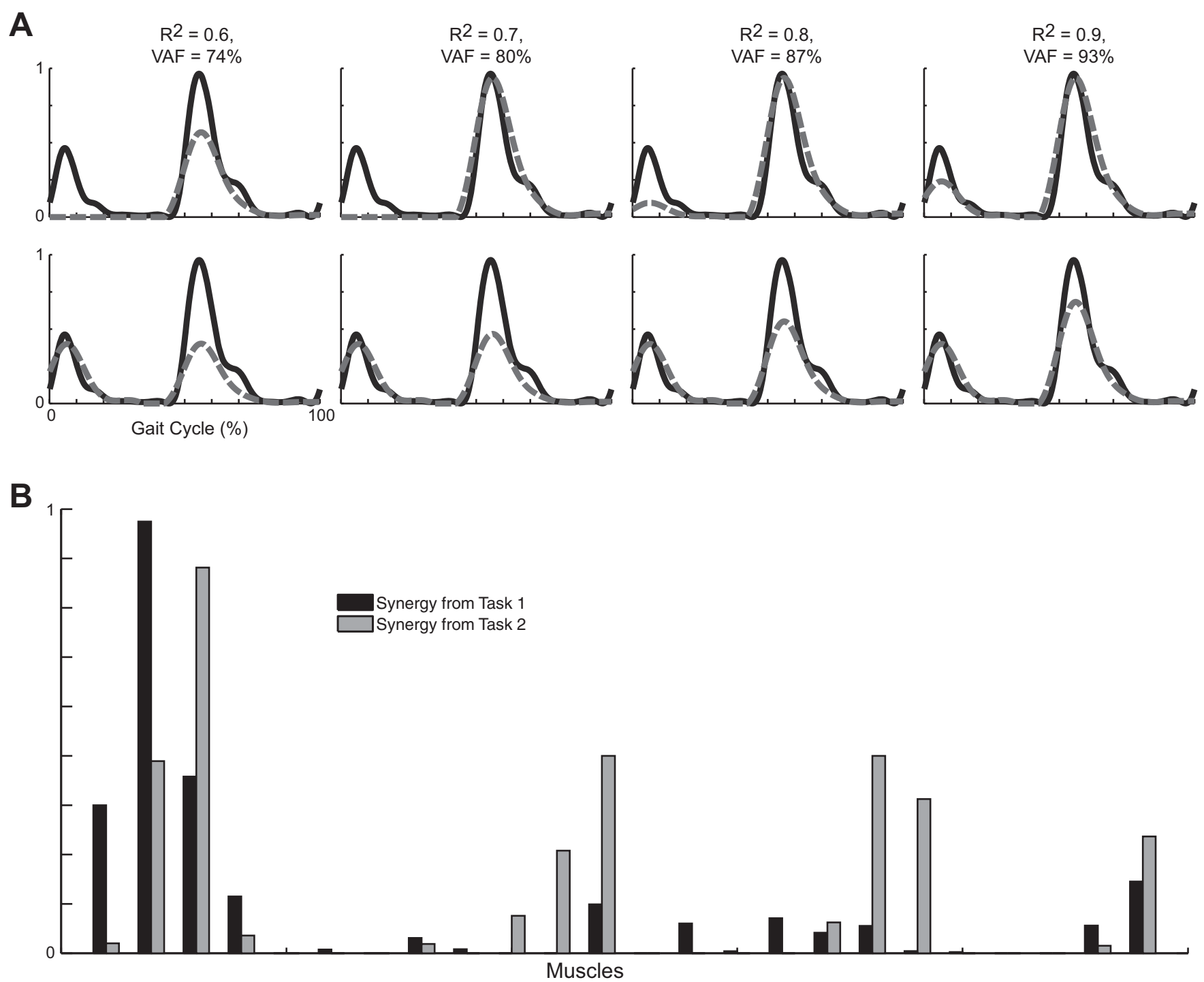

Fig. 10. Methodological considerations. A: selecting an EMG reconstruction criterion. Example reconstruction estimates (dashed gray line) of varying quality $\left(0.6 \leq R^{2} \leq 0.9\right.$ and $\left.74 \% \leq \mathrm{VAF} \leq 93 \%\right)$ are shown for a representative EMG envelope (solid black line). $R^{2}$ and variability accounted for (VAF) were computed via centered and uncentered Pearson correlation, respectively. Each column, from right to left, corresponds to decreasing quality of EMG reconstructions. The top row roughly corresponds to degrading the fit of the first EMG activation peak and the bottom row to degrading the second peak. We selected an Individual-Muscle evaluation criterion $\left(R^{2} \geq 0.8\right)$ to subjectively identify individual EMGs that were adequately reconstructed, considering the noise and variability inherent in surface EMG measurements. EMG estimates with correlations below this criterion tend to be missing important features of the muscle activity (e.g., the absence of first EMG peak in top row when $R^{2}<0.8$ ) or exhibit additional bursts of activity (not shown) that are inconsistent with empirical recordings. $B$ : identifying shared synergies. Muscle weightings (1-24) are depicted for hypothetical synergies from 2 different tasks (depicted as black and gray bars). Thus each pair of bars represents a weighting for the same muscle. According to conventional post hoc statistical techniques these synergies (which are moderately correlated with $R^{2}=0.27$ ) would be defined as the same, shared synergy, since they exhibit similarity above random chance (e.g., based on critical value of Pearson coefficient with significance level 0.01). However, trying to reconcile the differences between these synergies degrades their abilities to generate the measured muscle activity, thus undermining the proposed benefits of shared primitives. In this study we present an alternative method for identifying shared modules, which avoids this issue.

empirical EMG profiles observed during each task, thus undermining the original purpose of the shared primitives. In summary, correlating modules extracted from different tasks in order to identify the shared library (as opposed to testing the ability of the shared modules themselves to reconstruct muscle activity) likely overestimates the utility of the shared primitives.

\section{Conclusions}

We assessed simple modular strategies during multidirectional locomotion and introduced a new metric (the modular control ratio) to evaluate their abilities to simplify control. We found that modularity offers the potential to reduce dimensionality for individual tasks but that shared neural primitives have practical limitations in generating muscle activity for diverse locomotor behaviors. Thus, while it is conceivable that primitives adapted by task-specific sensory feedback or incorporated into a higher-dimensional control strategy might provide an effective basis for coordinating muscle activity, the challenge remains to identify more sophisticated, yet testable formulations of motor control that address these limitations. 


\section{ACKNOWLEDGMENTS}

The authors thank Andrea d'Avella for his insightful comments.

\section{GRANTS}

This work was supported in part by the Italian Ministry of Health (RC and RF grants), the Italian Ministry of University and Research (PRIN grant), the Italian Space Agency (DCMC and COREA grants), the European Union FP7-ICT program (MINDWALKER Grant no. 247959 and AMARSi Grant no. 248311), and the Whitaker International Program.

\section{DISCLOSURES}

No conflicts of interest, financial or otherwise, are declared by the author(s).

\section{AUTHOR CONTRIBUTIONS}

Author contributions: K.E.Z., V.L.S., Y.P.I., and F.L. conception and design of research; K.E.Z. and V.L.S. performed experiments; K.E.Z. analyzed data; K.E.Z., V.L.S., Y.P.I., and F.L. interpreted results of experiments; K.E.Z. prepared figures; K.E.Z. drafted manuscript; K.E.Z., V.L.S., Y.P.I., and F.L. edited and revised manuscript; K.E.Z., V.L.S., Y.P.I., and F.L. approved final version of manuscript.

\section{REFERENCES}

Alessandro C, Delis I, Nori F, Panzeri S, Berret B. Muscle synergies in neuroscience and robotics: from input-space to task-space perspectives. Front Comput Neurosci 7: 43, 2013.

d'Avella A, Bizzi E. Shared and specific muscle synergies in natural motor behaviors. Proc Natl Acad Sci USA 102: 3076-3081, 2005.

d'Avella A, Cesqui B, Lacquaniti F. Identifying muscle synergies from EMG decomposition: approaches, evidence, and potential application to neurorehabilitation [Online]. In: Converging Clinical and Engineering Research on Neurorehabilitation, edited by Pons JL, Torricelli D, Pajaro M. Berlin: Springer, 2013, p. 1243-1247. http://rd.springer.com/chapter/10.1007/9783-642-34546-3_207 [October 24, 2013].

d'Avella A, Saltiel P, Bizzi E. Combinations of muscle synergies in the construction of a natural motor behavior. Nat Neurosci 6: 300-308, 2003.

Berger DJ, Gentner R, Edmunds T, Pai DK, d'Avella A. Differences in adaptation rates after virtual surgeries provide direct evidence for modularity. J Neurosci 33: 12384-12394, 2013.

Bernstein N. The Co-ordination and Regulation of Movements. Oxford, UK: Pergamon, 1967.

Cappellini G, Ivanenko YP, Dominici N, Poppele RE, Lacquaniti F. Migration of motor pool activity in the spinal cord reflects body mechanics in human locomotion. J Neurophysiol 104: 3064-3073, 2010.

Cappellini G, Ivanenko YP, Poppele RE, Lacquaniti F. Motor patterns in human walking and running. J Neurophysiol 95: 3426-3437, 2006.

Cheung VC, d'Avella A, Tresch MC, Bizzi E. Central and sensory contributions to the activation and organization of muscle synergies during natural motor behaviors. J Neurosci 25: 6419-6434, 2005.

Chhabra M, Jacobs RA. Properties of synergies arising from a theory of optimal motor behavior. Neural Comput 18: 2320-2342, 2006.

Chvatal SA, Ting LH. Voluntary and reactive recruitment of locomotor muscle synergies during perturbed walking. J Neurosci 32: 12237-12250, 2012.

Clark DJ, Ting LH, Zajac FE, Neptune RR, Kautz SA. Merging of healthy motor modules predicts reduced locomotor performance and muscle coordination complexity post-stroke. J Neurophysiol 103: 844-857, 2010.

Courtine G, Harkema SJ, Dy CJ, Gerasimenko YP, Dyhre-Poulsen P. Modulation of multisegmental monosynaptic responses in a variety of leg muscles during walking and running in humans. J Physiol 582: 1125-1139, 2007.

Courtine G, Papaxanthis C, Schieppati M. Coordinated modulation of locomotor muscle synergies constructs straight-ahead and curvilinear walking in humans. Exp Brain Res 170: 320-335, 2006.

Delis I, Panzeri S, Pozzo T, Berret B. A unifying model of concurrent spatial and temporal modularity in muscle activity. J Neurophysiol 111: 675-693, 2014

Dominici N, Ivanenko YP, Cappellini G, d'Avella A, Mond $\mu$ V, Cicchese M, Fabiano A, Silei T, Paolo AD, Giannini C, Poppele RE, Lacquaniti
F. Locomotor primitives in newborn babies and their development. Science 334: 997-999, 2011.

Drew T, Kalaska J, Krouchev N. Muscle synergies during locomotion in the cat: a model for motor cortex control. J Physiol 586: 1239-1245, 2008.

Duysens J, Groote FD, Jonkers I. The flexion synergy, mother of all synergies and father of new models of gait. Front Comput Neurosci 7: 14, 2013.

Duysens J, van Wezel BM, van de Crommert HW, Faist M, Kooloos JG. The role of afferent feedback in the control of hamstrings activity during human gait. Eur J Morphol 36: 293-299, 1998.

Farina D, Merletti R, Enoka RM. The extraction of neural strategies from the surface EMG. J Appl Physiol 96: 1486-1495, 2004.

Flanders M. What is the biological basis of sensorimotor integration? Biol Cybern 104: 1-8, 2011.

Frere J, Hug F. Between-subject variability of muscle synergies during a complex motor skill. Front Comput Neurosci 6: 99, 2012.

Giszter SF, Hart CB, Silfies SP. Spinal cord modularity: evolution, development, and optimization and the possible relevance to low back pain in man. Exp Brain Res 200: 283-306, 2010.

Giszter S, Patil V, Hart C. Primitives, premotor drives, and pattern generation: a combined computational and neuroethological perspective. Prog Brain Res 165: 323-346, 2007.

Grasso R, Ivanenko YP, Zago M, Molinari M, Scivoletto G, Lacquaniti F. Recovery of forward stepping in spinal cord injured patients does not transfer to untrained backward stepping. Exp Brain Res 157: 377-382, 2004.

Gray EG, Basmajian JV. Electromyography and cinematography of leg and foot ("normal" and flat) during walking. Anat Rec 161: 1-15, 1968.

Grey MJ, Ladouceur M, Andersen JB, Nielsen JB, Sinkjær T. Group II muscle afferents probably contribute to the medium latency soleus stretch reflex during walking in humans. $J$ Physiol 534: 925-933, 2001.

Grillner S. Control of locomotion in bipeds, tetrapods, and fish. Compr Physiol 2011 (Online), Supplement 2: Handbook of Physiology, The Nervous System, Motor Control: 1179-1236 (First published in print 1981).

Hart CB, Giszter SF. A neural basis for motor primitives in the spinal cord. J Neurosci 30: 1322-1336, 2010.

Ivanenko YP, Cappellini G, Dominici N, Poppele RE, Lacquaniti F. Coordination of locomotion with voluntary movements in humans. $\mathrm{J} \mathrm{Neu}$ rosci 25: 7238-7253, 2005.

Ivanenko YP, Cappellini G, Poppele RE, Lacquaniti F. Spatiotemporal organization of $\alpha$-motoneuron activity in the human spinal cord during different gaits and gait transitions. Eur J Neurosci 27: 3351-3368, 2008.

Ivanenko YP, Poppele RE, Lacquaniti F. Five basic muscle activation patterns account for muscle activity during human locomotion. $J$ Physiol 556: 267-282, 2004.

Ivanenko YP, Poppele RE, Lacquaniti F. Motor control programs and walking. Neuroscientist 12: 339-348, 2006.

Jacobs RA, Jordan MI. Learning piecewise control strategies in a modular neural network architecture. IEEE Trans Syst Man Cybern 23: 337-345, 1993.

Kargo WJ, Giszter SF. Individual premotor drive pulses, not time-varying synergies, are the units of adjustment for limb trajectories constructed in spinal cord. $J$ Neurosci 28: 2409-2425, 2008.

Kargo WJ, Ramakrishnan A, Hart CB, Rome LC, Giszter SF. A simple experimentally based model using proprioceptive regulation of motor primitives captures adjusted trajectory formation in spinal frogs. J Neurophysiol 103: 573-590, 2010.

Kendall FP. Muscles: Testing and Function with Posture and Pain. Baltimore, MD: Lippincott Williams \& Wilkins, 2005.

Kutch JJ, Kuo AD, Bloch AM, Rymer WZ. Endpoint force fluctuations reveal flexible rather than synergistic patterns of muscle cooperation. $J$ Neurophysiol 100: 2455-2471, 2008.

Kutch JJ, Valero-Cuevas FJ. Challenges and new approaches to proving the existence of muscle synergies of neural origin. PLoS Comput Biol 8: e1002434, 2012.

Lacquaniti F, Ivanenko YP, d'Avella A, Zelik KE, Zago M. Evolutionary and developmental modules. Front Comput Neurosci 7: 61, 2013.

Lacquaniti F, Ivanenko YP, Zago M. Patterned control of human locomotion. J Physiol 590: 2189-2199, 2012.

Lamb T, Yang JF. Could different directions of infant stepping be controlled by the same locomotor central pattern generator? J Neurophysiol 83: 2814-2824, 2000.

Lee DD, Seung HS. Learning the parts of objects by non-negative matrix factorization. Nature 401: 788-791, 1999. 
Lewicki MS, Sejnowski TJ. Learning overcomplete representations. Neural Comput 12: 337-365, 2000.

Llinás RR, Roy S. The "prediction imperative" as the basis for self-awareness. Philos Trans R Soc B 364: 1301-1307, 2009.

Macpherson JM. How flexible are muscle synergies? In: Motor Control: Concepts and Issues, edited by Humphrey DR, Freund HJ. New York: Wiley, 1991, p. 33-47.

McCrea DA, Rybak IA. Modeling the mammalian locomotor CPG: insights from mistakes and perturbations. Prog Brain Res 165: 235-253, 2007.

McCrea DA, Rybak IA. Organization of mammalian locomotor rhythm and pattern generation. Brain Res Rev 57: 134-146, 2008.

McKay JL, Ting LH. Functional muscle synergies constrain force production during postural tasks. J Biomech 41: 299-306, 2008.

Mussa-Ivaldi FA. Modular features of motor control and learning. Curr Opin Neurobiol 9: 713-717, 1999.

Overduin SA, d'Avella A, Carmena JM, Bizzi E. Microstimulation activates a handful of muscle synergies. Neuron 76: 1071-1077, 2012.

Overduin SA, d'Avella A, Roh J, Bizzi E. Modulation of muscle synergy recruitment in primate grasping. J Neurosci 28: 880-892, 2008.

Patla AE, Calvert TW, Stein RB. Model of a pattern generator for locomotion in mammals. Am J Physiol Regul Integr Comp Physiol 248: R484R494, 1985.

Perry J. Ankle foot complex. In: Gait Analysis: Normal and Pathological Function. Thorofare, NJ: Slack, 1992, p. 51-88.

Reeser LA, Susman RL, Stern JT. Electromyographic studies of the human foot: experimental approaches to hominid evolution. Foot Ankle Int 3: 391-407, 1983.

Roh J, Rymer WZ, Beer RF. Robustness of muscle synergies underlying three-dimensional force generation at the hand in healthy humans. $J$ Neurophysiol 107: 2123-2142, 2012.

De Rugy A, Loeb GE, Carroll TJ. Muscle coordination is habitual rather than optimal. J Neurosci 32: 7384-7391, 2012.

De Rugy A, Loeb GE, Carroll TJ. Are muscle synergies useful for neural control? Front Comput Neurosci 7: 19, 2013.

Safavynia SA, Ting LH. Task-level feedback can explain temporal recruitment of spatially fixed muscle synergies throughout postural perturbations. J Neurophysiol 107: 159-177, 2012.

Santello M, Flanders M, Soechting JF. Postural hand synergies for tool use. J Neurosci 18: 10105-10115, 1998.
Saraswat P, Andersen MS, Macwilliams BA. A musculoskeletal foot model for clinical gait analysis. J Biomech 43: 1645-1652, 2010.

Sinkjær T, Andersen JB, Ladouceur M, Christensen LO, Nielsen JB. Major role for sensory feedback in soleus EMG activity in the stance phase of walking in man. $J$ Physiol 523: 817-827, 2000.

Soechting JF, Lacquaniti F. An assessment of the existence of muscle synergies during load perturbations and intentional movements of the human arm. Exp Brain Res 74: 535-548, 1989.

Steele K, Tresch MC, Perreault EJ. Using musculoskeletal modeling and simulation to investigate the accuracy and reliability of muscle synergies (Abstract). 23rd Neural Control of Movement Proceedings, 2013

Stein PS. Motor pattern deletions and modular organization of turtle spinal cord. Brain Res Rev 57: 118-124, 2008.

Tessitore G, Sinigaglia C, Prevete R. Hierarchical and multiple hand action representation using temporal postural synergies. Exp Brain Res 225: 11-36, 2013.

Ting LH. Dimensional reduction in sensorimotor systems: a framework for understanding muscle coordination of posture. Prog Brain Res 165: 299321, 2007.

Ting LH, Macpherson JM. A limited set of muscle synergies for force control during a postural task. J Neurophysiol 93: 609-613, 2005.

Todorov E. Optimality principles in sensorimotor control. Nat Neurosci 7: 907-915, 2004.

Torres-Oviedo G, Macpherson JM, Ting LH. Muscle synergy organization is robust across a variety of postural perturbations. J Neurophysiol 96: 1530-1546, 2006.

Tresch MC, Jarc A. The case for and against muscle synergies. Curr Opin Neurobiol 19: 601-607, 2009.

Tresch MC, Saltiel P, Bizzi E. The construction of movement by the spinal cord. Nat Neurosci 2: 162-167, 1999.

Wakeling JM, Horn T. Neuromechanics of muscle synergies during cycling. J Neurophysiol 101: 843-854, 2009.

Winter DA, Yack HJ. EMG profiles during normal human walking: strideto-stride and inter-subject variability. Electroencephalogr Clin Neurophysiol 67: 402-411, 1987.

Zehr EP. Neural control of rhythmic human movement: the common core hypothesis. Exerc Sport Sci Rev 33: 54-60, 2005. 